\title{
CALCIUM-RICH GAP TRANSIENTS IN THE REMOTE OUTSKIRTS OF GALAXIES
}

\author{
Mansi M. Kasliwal ${ }^{1,2}$, S. R. Kulkarni ${ }^{1}$, Avishay Gal-Yam ${ }^{3}$, Peter E. Nugent ${ }^{4,5}$, Mark Sullivan $^{6}$, Lars Bildsten $^{7,8}$, \\ Ofer Yaron $^{3}$, Hagai B. Perets ${ }^{9}$, Iair Arcavi ${ }^{3}$, Sagi Ben-Ami ${ }^{3}$, Varun B. Bhalerao ${ }^{1}$, Joshua S. Bloom ${ }^{5}$, \\ S. Bradley Cenko ${ }^{5}$, Alexei V. Filippenko ${ }^{5}$, Dale A. Frail ${ }^{10}$, Mohan Ganeshalingam ${ }^{5}$, Assaf Horesh ${ }^{1}$, \\ D. Andrew Howell ${ }^{8,11}$, Nicholas M. Law ${ }^{12}$, Douglas C. Leonard ${ }^{13}$, Weidong Li ${ }^{5}$, Eran O. OfeK ${ }^{3}$, David Polishook ${ }^{3}$, \\ Dovi Poznanski ${ }^{14}$, Robert M. Quimby ${ }^{1,15}$, Jeffrey M. Silverman ${ }^{5}$, Assaf Sternberg $^{3}$, And Dong Xu $^{3}$ \\ ${ }^{1}$ Cahill Center for Astrophysics, California Institute of Technology, Pasadena, CA 91125, USA \\ ${ }^{2}$ Observatories of the Carnegie Institution for Science, 813 Santa Barbara St, Pasadena, CA 91101, USA \\ ${ }^{3}$ Benoziyo Center for Astrophysics, Faculty of Physics, The Weizmann Institute of Science, Rehovot 76100, Israel \\ ${ }^{4}$ Computational Cosmology Center, Lawrence Berkeley National Laboratory, 1 Cyclotron Road, Berkeley, CA 94720, USA \\ ${ }^{5}$ Department of Astronomy, University of California, Berkeley, CA 94720-3411, USA \\ ${ }^{6}$ Department of Physics, Oxford University, Oxford OX1 3RH, UK \\ ${ }^{7}$ Department of Physics, University of California, Santa Barbara, CA 93106, USA \\ ${ }^{8}$ Kavli Institute for Theoretical Physics, University of California, Santa Barbara, CA 93106, USA \\ ${ }_{9}^{9}$ Harvard-Smithsonian Center for Astrophysics, 60 Garden St., Cambridge, MA 02338, USA \\ ${ }^{10}$ National Radio Astronomy Observatory, Array Operations Center, Socorro, NM 87801, USA \\ ${ }^{11}$ Las Cumbres Observatory Global Telescope Network, Inc., Santa Barbara, CA 93117, USA \\ 12 Dunlap Institute for Astronomy and Astrophysics, University of Toronto, 50 St. George Street, Toronto M5S 3H4, Ontario, Canada \\ ${ }^{13}$ Department of Astronomy, San Diego State University, San Diego, CA 92182, USA \\ ${ }^{14}$ School of Physics and Astronomy, Tel-Aviv University, Tel Aviv 69978, Israel \\ ${ }^{15}$ IPMU, University of Tokyo, Kashiwanoha 5-1-5, Kashiwa-shi, Chiba, Japan \\ Received 2011 November 28; accepted 2012 June 19; published 2012 August 7
}

\begin{abstract}
From the first two seasons of the Palomar Transient Factory, we identify three peculiar transients (PTF 09dav, PTF 10iuv, and PTF 11bij) with five distinguishing characteristics: peak luminosity in the gap between novae and supernovae $\left(M_{R} \approx-15.5\right.$ to $\left.-16.5 \mathrm{mag}\right)$, rapid photometric evolution $\left(t_{\text {rise }} \approx 12-15\right.$ days $)$, large photospheric velocities $\left(\approx 6000-11,000 \mathrm{~km} \mathrm{~s}^{-1}\right)$, early spectroscopic evolution into nebular phase $(\approx 1-3$ months $)$, and peculiar nebular spectra dominated by calcium. We also culled the extensive decade-long Lick Observatory Supernova Search database and identified an additional member of this group, SN 2007ke. Our choice of photometric and spectroscopic properties was motivated by SN 2005E (Perets et al.). To our surprise, as in the case of SN 2005E, all four members of this group are also clearly offset from the bulk of their host galaxy. Given the well-sampled early- and late-time light curves, we derive ejecta masses in the range of 0.4-0.7 $M_{\odot}$. Spectroscopically, we find that there may be a diversity in the photospheric phase, but the commonality is in the unusual nebular spectra. Our extensive follow-up observations rule out standard thermonuclear and standard core-collapse explosions for this class of "calcium-rich gap" transients. If the progenitor is a white dwarf, we are likely seeing a detonation of the white dwarf core and perhaps even shock-front interaction with a previously ejected nova shell. If the progenitor is a massive star, a nonstandard channel specific to a low-metallicity environment needs to be invoked (e.g., ejecta fallback leading to black hole formation). Detection (or the lack thereof) of a faint underlying host (dwarf galaxy and cluster) will provide a crucial and decisive diagnostic to choose between these alternatives.
\end{abstract}

Key words: galaxies: clusters: general - Galaxy: halo - novae, cataclysmic variables - supernovae: individual (SN2005E, SN2007ke, PTF09dav, PTF10iuv, PTF11bij) - surveys - white dwarfs

Online-only material: color figures

\section{INTRODUCTION}

In the past decade, supernova surveys targeting nearby, luminous galaxies have been immensely successful since the total local starlight searched is significantly larger than in untargeted pointings of equal area. The Palomar Transient Factory (PTF; Law et al. 2009; Rau et al. 2009) ${ }^{16}$ is a wideangle transient survey that has an ongoing Dynamic Cadence experiment that combines the advantages of both a targeted and an untargeted survey. This experiment searches wide-angle pointings of local $(d<200 \mathrm{Mpc})$ galaxy light concentrations at a one-day cadence to a median depth of $21 \mathrm{mag}$. The depth, cadence, and choice of pointings allow us to find transients

\footnotetext{
${ }^{16} \mathrm{http}: / / \mathrm{www} . a s t r o . c a l t e c h . e d u / p t f /$
}

that are fainter, faster, and rarer than supernovae. Furthermore, these survey design characteristics facilitate the discovery of intracluster transients as well as transients in the far-flung outskirts of their host galaxies.

While monitoring supernovae from the targeted Lick Observatory Supernova Search (LOSS; Li et al. 2000; Filippenko et al. 2001) with the Katzman Automatic Imaging Telescope (KAIT), Filippenko et al. (2003) noted that there was a subclass of peculiar Type I supernovae that appeared to be rich in calcium at an early stage. Perets et al. (2010) presented extensive observations of one of these events, SN 2005E. In addition to the calcium-richness, SN 2005E was subluminous and rapidly evolving. Furthermore, SN 2005E was located $23 \mathrm{kpc}$ away from the nucleus of its host galaxy, with no signs of underlying star formation to deep limits. The physical origin of SN 2005E remains a matter of debate. 

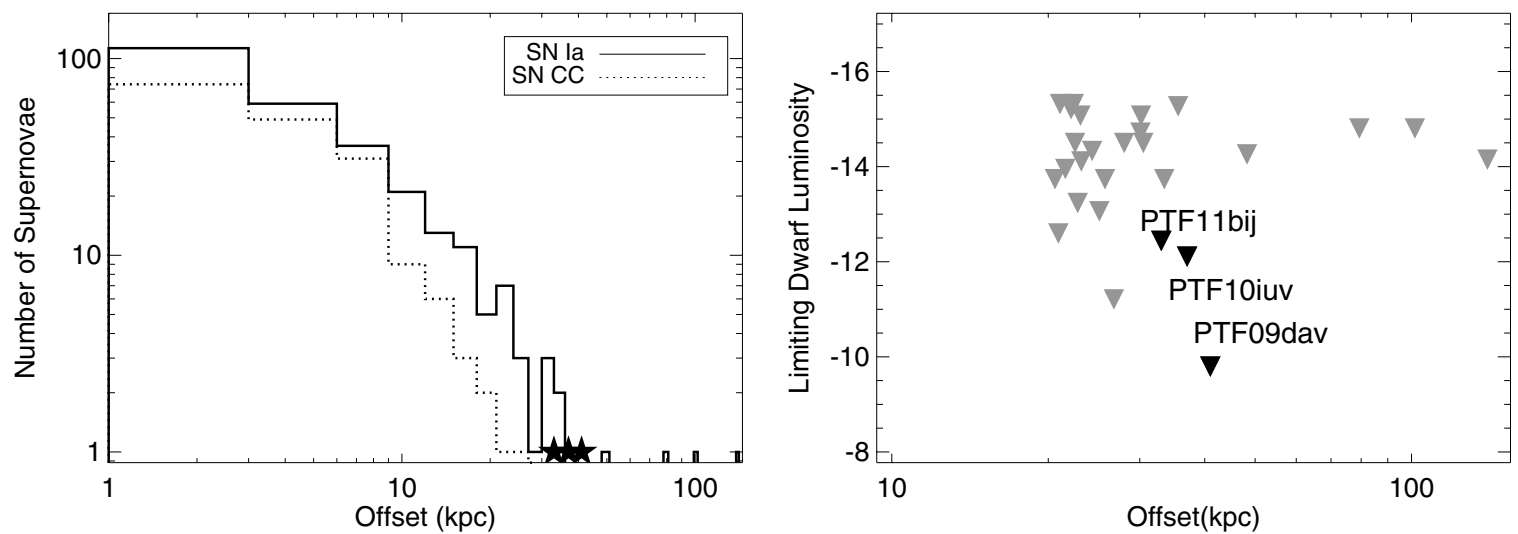

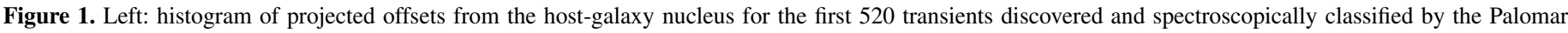

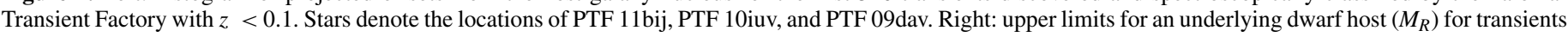
with offset greater than $20 \mathrm{kpc}$.

Motivated thus, and given that the survey design of PTF allows it be to especially sensitive to find and follow up such events, we searched the PTF discovery stream. We find three transients-PTF 09dav, PTF 10iuv, and PTF 11bij-that share five distinguishing characteristics with SN 2005E: (1) peak luminosity intermediate between that of novae and supernovae, (2) faster photometric evolution (rise and decline) than supernovae, (3) photospheric velocities comparable to supernovae, (4) early evolution to the nebular phase, and (5) nebular spectra dominated by calcium emission. These five explosion properties define a distinct class of "calcium-rich gap" transients. (We closely scrutinize the choice of this set of explosion characteristics in Section 7.1.)

Remarkably, these three PTF transients were also found in remote locations, more than $30 \mathrm{kpc}$ away from the nuclei of their host galaxies. Location was not used as a selection criterion; yet, we do not see any other PTF supernovae thus far that display the five explosion characteristics listed above (see Section 7.2 for details). Therefore, in Section 2 we take a closer look at the locations of these three transients in the context of locations of other PTF supernovae.

The rest of the paper is organized as follows. We present observations of PTF 09dav, PTF 10iuv, and PTF 11bij in Sections 3, 4, and 5, respectively. We show the available data on the six archival candidates from the past decade in Section 6. We analyze the properties of the combined sample of well-observed transients in this class in Section 7. We discuss whether this class has an underlying physical commonality and speculate on its origin in Section 8, and conclude in Section 9.

\section{OFFSET DISTRIBUTION OF PTF SUPERNOVAE}

The location of a transient explosion has long been exploited as a clue to determining its nature. It has been suggested that the classical nova population is bimodal, with some dependence on whether the nova is located in the Galactic disk or bulge (Shafter et al. 2011). Several studies of supernova host-galaxy properties as well as the site of the supernova within the host galaxy have been undertaken (Howell 2001; van den Bergh 1997; Prieto et al. 2008; Hakobyan et al. 2009; Boissier \& Prantzos 2009; Anderson \& James 2009). Subluminous Type Ia supernovae (SNe Ia) are found preferentially in old environments (Howell 2001; for a review of spectroscopic classification of supernovae, see Filippenko 1997). Core-collapse supernovae (SNe CC) and more luminous SNe Ia are preferentially found in late-type galaxies (Hamuy et al. 1996). Type Ic supernovae are not found in dwarfs (Arcavi et al. 2010) and SNe Ibc are more centrally concentrated than SNe II (Anderson \& James 2009). About 15\% of the stellar mass is expected to be in the intergalactic medium in clusters, and a handful of candidate intracluster supernovae have been discovered (Gal-Yam et al. 2003; Sand et al. 2011). At higher energies, gamma-ray burst offsets from host galaxies offered a clue to their progenitors (Fruchter et al. 2006; Bloom et al. 2006).

In the first two years, PTF discovered and spectroscopically confirmed over 1300 extragalactic transients. Here, we limit the study of the offset distribution of PTF supernovae to the first 520 transients which were nearby, with redshift $z<0.1$. We compute precise projected offsets from the host galaxies for each transient, and the resulting histogram is shown in Figure 1. The redshift of the host galaxy is consistent with the redshift of the transient. Note that the offset is projected and hence, only a lower limit.

We split the population into $\mathrm{SNe} \mathrm{CC}$ and thermonuclear supernovae. We find that the core-collapse and thermonuclear populations show similar distributions out to a projected offset from the host nucleus of $\approx 9 \mathrm{kpc}$ suggesting that the average distribution is proportional to starlight. Beyond $9 \mathrm{kpc}$, the thermonuclear population shows a heavily extended tail suggesting a second parameter governing their rate or perhaps even two progenitor populations (Mannucci et al. 2006). A cautionary note here is that although this sample is relatively more homogeneous in that it is drawn from a single survey with extensive follow-up observations, it is not spared the vagaries of incompleteness due to weather and follow-up bias.

Next, we take a closer look at the population with offsets larger than $20 \mathrm{kpc}$. We co-add available pre-explosion data to derive limiting magnitudes on a satellite host galaxy at the location of the supernova (see Figure 1 (right panel)). The redshift limit constrains the luminosity of underlying host galaxies based on our pre-explosion co-adds to at least -16 mag.

Focusing on the dozen transients with offsets larger than $30 \mathrm{kpc}$, we find nine $\mathrm{SNe} \mathrm{Ia}$, zero $\mathrm{SNe} \mathrm{CC}$, and three peculiar transients. Five SNe Ia-PTF 09cex, PTF 10fjg, PTF 10xua, PTF 10xgb, and PTF 10xgc-with offsets of 30-36 kpc have spectroscopically confirmed host redshifts. Three SN Ia-PTF 10qnk (101 kpc), PTF 10qht (79 kpc), and PTF 10qyx (48 kpc)-are possibly intracluster supernovae (A. Horesh et al., in preparation). The furthest offset $\mathrm{SNe}$ Ia, 


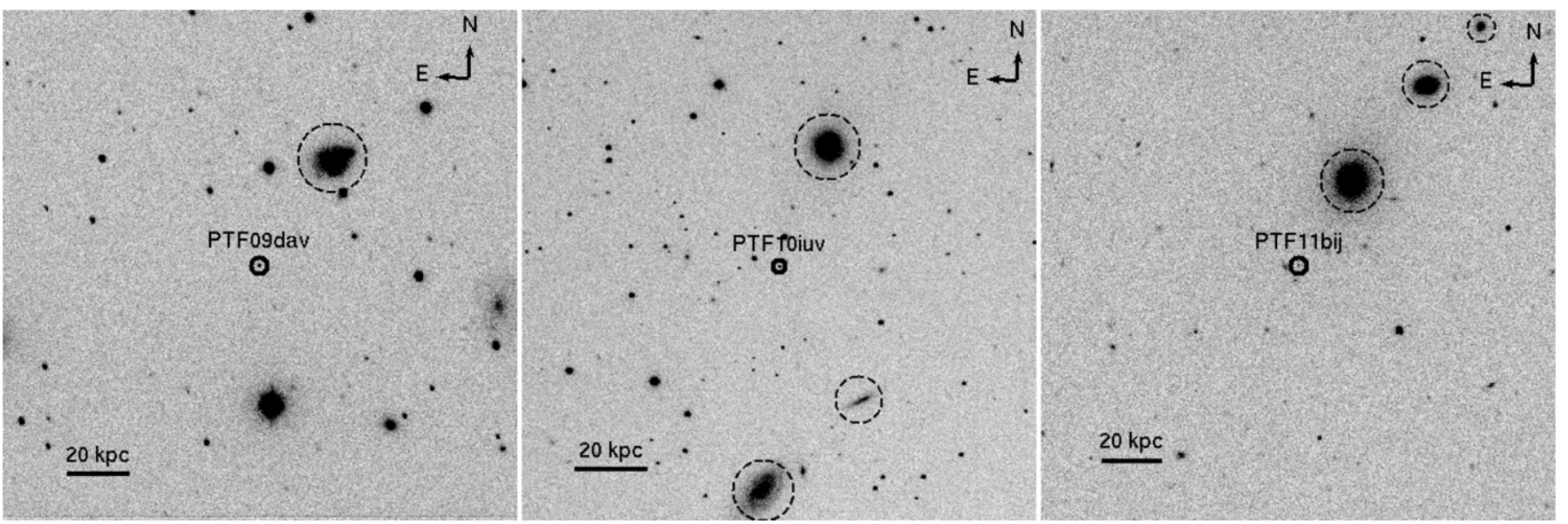

Figure 2. Left: PTF 09dav is offset from its late-type host (circled) by $40 \mathrm{kpc}$. Note that the galaxy on the western edge is unrelated as it is at a different redshift. Center: PTF 10iuv is offset from a galaxy group (circled) with early-type and late-type galaxies; the nearest potential host is $37 \mathrm{kpc}$ away. Right: PTF $11 \mathrm{bij}$ is offset from a group of early-type galaxies (circled); the nearest is $33 \mathrm{kpc}$ away.

PTF10ops (140 kpc), has peculiar properties and is the subject of another paper (Maguire et al. 2011).

The three peculiar transients are the topic of this paper. PTF 09dav is offset from a spiral host by $40 \mathrm{kpc}$; PTF 10iuv is in a galaxy cluster containing early- and late-type galaxies, with the closest being an elliptical galaxy $37 \mathrm{kpc}$ away; and PTF 11bij is in a cluster of early-type galaxies, with the closest being $33 \mathrm{kpc}$ away (Figure 2). Note that in units of host-galaxy Petrosian radii, these offsets correspond to 4.9, 5.9, and 3.8 radii, respectively. We cannot rule out the possibility of an underlying low-luminosity dwarf host; we can only constrain dwarfs to a limiting $R$-band absolute magnitude of $-9.8,-12.1$, and -12.4 for PTF 09dav, PTF 10iuv, and PTF 11bij, respectively. Finding dwarf satellites at a separation of $40 \mathrm{kpc}$ would not be unusual (cf. Andromeda's $-8.5 \mathrm{mag}$ satellite at $350 \mathrm{kpc}$; Slater et al. 2011).

\section{OBSERVATIONS: PTF 09DAV}

We presented the discovery, light curve, and photospheric spectra of PTF 09dav in a previous paper (Sullivan et al. 2011). We found that PTF 09dav had a low peak absolute magnitude of $M_{R} \approx-16.3$ and a short rise time of 12 days relative to SNe Ia (which peak at -19 mag with a rise-time of 17.5 days). We also found that the photospheric spectra resembled those of SN 1991bg-like subluminous SNe Ia but with very unusual strong lines of Sc II, Mg I, and possibly Sr II. Photospheric velocities were $\approx 6000 \mathrm{~km} \mathrm{~s}^{-1}$. Here, we present late-time imaging and nebular spectroscopy of PTF09dav.

\subsection{Late-time Imaging}

We undertook deep imaging in the $g$-band and $R$-band filters at the position of PTF 09dav with the Low Resolution Imaging Spectrometer (LRIS; Oke et al. 1995) on the Keck I telescope on 2010 May 15.603 and July 9.584 (UT dates are used throughout this paper), 9-11 months after explosion. We registered these images with a Palomar 60 inch (P60) image of PTF 09dav. No source is detected to a $3 \sigma$ limiting magnitude of $R=26.2 \mathrm{mag}$ (Table 1 and Figure 3). This constrains any satellite, dwarf host to be fainter than $M_{R}=-9.8$ mag.

We undertook imaging in the $K^{\prime}$ band with Laser Guide Star Adaptive Optics (Wizinowich et al. 2006; van Dam et al. 2006) on the Keck II telescope with the Near Infrared Camera 2 (NIRC2). On 2010 June 17.568, we obtained 10 images of $10 \mathrm{~s}$



Figure 3. Deep late-time $g$-band imaging with Keck I/LRIS showing no host galaxy at the position of PTF 09dav brighter than 26 mag. The registration accuracy is $0{ }^{\prime \prime} 241$. We denote the position of PTF 09dav with a $5 \sigma$ position error circle.

Table 1

Late-time Observations PTF 09dav

\begin{tabular}{lccccc}
\hline \hline $\begin{array}{l}\text { Date } \\
\text { (UT 2010) }\end{array}$ & $\begin{array}{l}\text { Phase } \\
\text { (day) }\end{array}$ & Facility & $\begin{array}{c}\text { Exposure } \\
(\mathrm{s})\end{array}$ & Filter & Magnitude \\
\hline May 15.612 & 279.7 & Keck I/LRIS & 1230 & $g$ & $>26.0$ \\
May 15.614 & 279.7 & Keck I/LRIS & 1030 & $R$ & $>24.8$ \\
June 17.568 & 313.7 & Keck II/NIRC2 & 100 & $K^{\prime}$ & $>21.1$ \\
July 9.584 & 334.7 & Keck I/LRIS & 1450 & $g$ & $>25.4$ \\
July 9.584 & 334.7 & Keck I/LRIS & 1200 & $R$ & $>26.2$ \\
\hline
\end{tabular}

co-added integrations. The zero point was derived relative to the Two Micron All Sky Survey catalog (Skrutskie et al. 2006). No source was detected to a $3 \sigma$ limit of $K^{\prime}=21.1 \mathrm{mag}$.

\subsection{Nebular Spectroscopy}

On 2009 November 11, only three months after maximum light, a spectrum taken with LRIS on Keck I revealed that 
Table 2

Lines in Nebular Spectra

\begin{tabular}{lccccrr}
\hline \hline Transient & Ion & $\begin{array}{c}\text { Line Center } \\
(\AA)\end{array}$ & $\begin{array}{c}\text { Shift }^{\mathrm{a}} \\
\left(\mathrm{km} \mathrm{s}^{-1}\right)\end{array}$ & $\begin{array}{c}\text { Flux } \\
\left(\mathrm{erg} \mathrm{cm}^{-2} \mathrm{~s}^{-1}\right)\end{array}$ & $\begin{array}{c}\text { Width }^{\mathrm{b}} \\
(\AA)\end{array}$ & $\begin{array}{c}\text { Velocity Width } \\
\left(\mathrm{km} \mathrm{s}^{-1}\right)\end{array}$ \\
\hline SN 2005E & O I & 6360.6 & -1120 & $2.0 \times 10^{-15}$ & 107.8 & 5110 \\
SN 2005E & {$[\mathrm{Ca}$ II $]$} & 7367.0 & -17 & $1.5 \times 10^{-14}$ & 127.6 & 5240 \\
SN 2005E & Ca II & 8710.4 & 2530 & $2.7 \times 10^{-14}$ & 299.0 & 10470 \\
PTF 09dav & H $\alpha$ & 6820.4 & 840 & $7.6 \times 10^{-17}$ & 23.5 & 1000 \\
PTF 09dav & {$[$ Ca II } & 7579.1 & 250 & $2.0 \times 10^{-15}$ & 166.1 & 6600 \\
PTF 10iuv & O I & 6467.7 & -460 & $1.7 \times 10^{-16}$ & 170.8 & 8090 \\
PTF 10iuv & [Ca II & 7465.4 & -410 & $8.0 \times 10^{-16}$ & 134.2 & 5510 \\
PTF 10iuv & Ca II & 8827.3 & 2150 & $9.4 \times 10^{-16}$ & 312.8 & 10950 \\
PTF 11bij & O I & 6531.5 & -1010 & $8.6 \times 10^{-17}$ & 98.8 & 4680 \\
PTF 11bij & {$[$ Ca II $]$} & 7573.5 & 406 & $5.8 \times 10^{-16}$ & 136.9 & 5620 \\
PTF 11bij & Ca II & 8960.0 & 3140 & $1.3 \times 10^{-15}$ & 257.0 & 9000 \\
\hline
\end{tabular}

Notes.

${ }^{\text {a }}$ Shift is computed relative to the velocity of the putative host galaxy.

b FWHM.

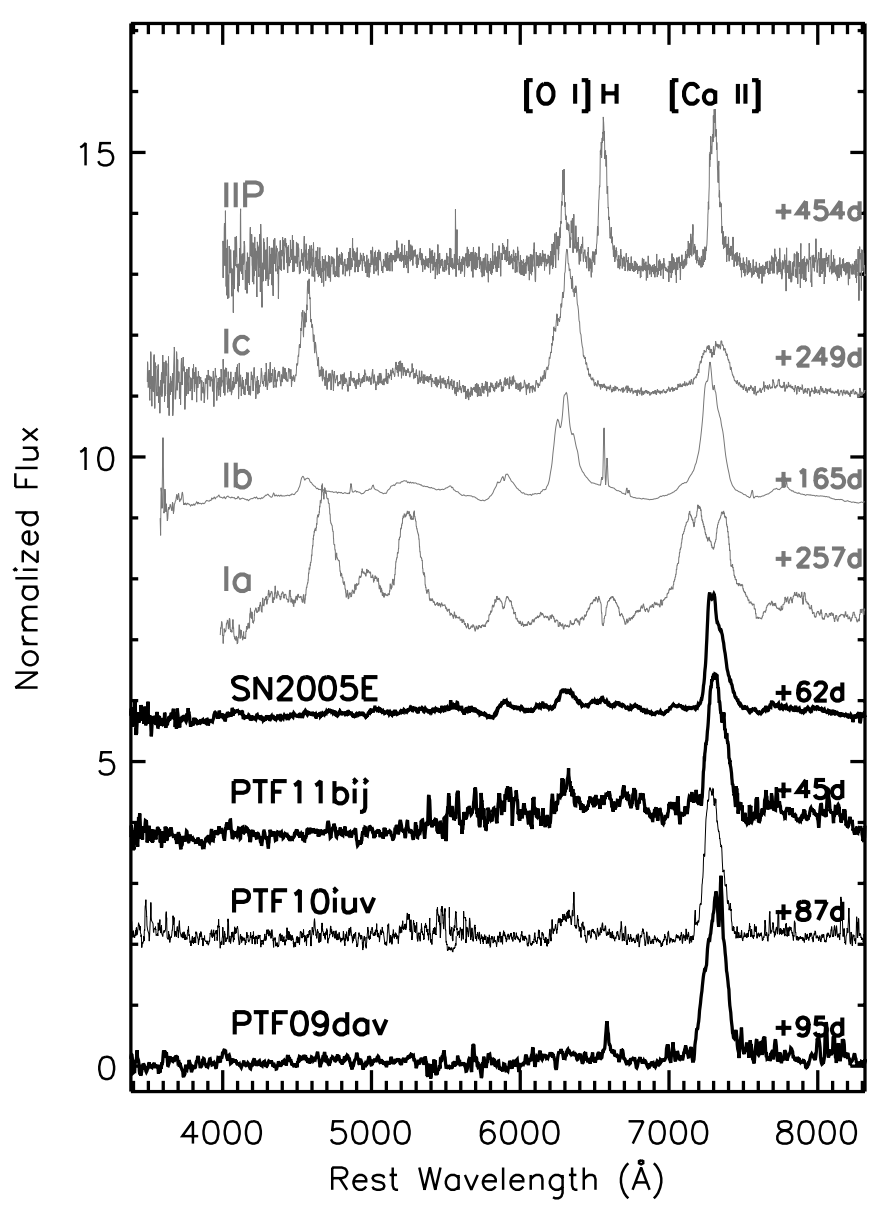

Figure 4. Nebular spectra of PTF 09dav, PTF 10iuv, PTF 11bij, and SN 2005E. All four are very calcium-rich. Also shown for comparison, nebular spectra of an SN Ia (SN 1986G, +257 day; Asiago Catalog), an SN Ib (SN 2007C, +165 day; Taubenberger et al. 2009), an SN Ic (SN 2002ap, +249 day; Gal-Yam et al. 2002), and an SN IIP (SN 2004et, +454 day; Sahu et al. 2006). The nebular spectra of SNe Ia are dominated by [Fe II], [Fe III], and [Co III] emission lines. The nebular spectra of SNe CC typically have prominent hydrogen or a much lower ratio of calcium to oxygen.

PTF 09dav had become nebular. The timescale to become nebular was surprising as it was faster than that of typical supernovae by a factor of a few. Furthermore, only two emission features are seen- $-\mathrm{H} \alpha$ and [Ca II] $\lambda \lambda$ 7291, 7324 (Figure 4). The width, redshift, and flux of the lines are summarized in Table 2. The absence of the Ca II near-infrared (IR) triplet $(\lambda \lambda 8498$, $8542,8662)$ is indicative of a low circumstellar density. No $\mathrm{H} \alpha$ was seen in the photospheric spectra presented by Sullivan et al. (2011). The presence of $\mathrm{H} \alpha$ emission is indicative of a photoionized circumburst medium, suggesting possible interaction with stellar wind. Neither oxygen (prominent in normal SN CC) nor iron lines (prominent in SNe Ia) are seen in the nebular phase. These characteristics of the nebular spectrum were unprecedented and no match could be found in supernova libraries.

\section{OBSERVATIONS: PTF 10IUV}

\subsection{Discovery and Light Curve}

On 2010 May 31.241, PTF discovered a new transient, PTF 10iuv, at $\alpha(\mathrm{J} 2000)=17^{\mathrm{h}} 16^{\mathrm{m}} 54^{\mathrm{s}} .27$ and $\delta(\mathrm{J} 2000)=$ $+31^{\circ} 33^{\prime} 51^{\prime \prime}$.7. It was initially found at $R=21.2 \mathrm{mag}$, and we monitored its brightness with the P60 in the Bgriz filters for three months (Cenko et al. 2006). Late-time photometric observations were taken with the Large Format Camera (LFC) on the Palomar 200 inch telescope and LRIS on the Keck I telescope.

Data were reduced following standard procedures and aperture photometry was performed. Photometric calibration was done relative to photometry of field stars from the Sloan Digital Sky Survey (Abazajian et al. 2009). A common set of calibration stars was chosen for the P48, P60, LFC, and LRIS data for consistency. Conversion from ugriz to the $B$ band was done following Jordi et al. (2006).

The light curve is summarized in Figure 5 and Table 3. PTF 10iuv peaked on June 10 with $r=19.0$ mag. In $r$ band, it rapidly rose by 2 mag in 10 days, followed by a rapid decline at the rate of 1 mag in 12 days. After a month, PTF 10iuv evolved slowly at the rate of $0.02 \mathrm{mag} \mathrm{day}^{-1}$ for three months followed by 0.005 mag day $^{-1}$. The color was neither extremely red nor blue. It evolved from $g-i \approx 0.4$ near maximum to $g-i \approx 0.7$ one month later.

\subsection{Spectroscopy}

On June 7, we obtained a classification spectrum using ISIS on the William Herschel Telescope (WHT). Subsequently, we continued to monitor the evolution with the Keck I (+LRIS) 
Table 3

Optical Light Curves

\begin{tabular}{|c|c|c|c|}
\hline MJD & Filter & Mag & Facility \\
\hline \multicolumn{4}{|l|}{ PTF10iuv } \\
\hline 55335.450 & $r$ & $>21.13$ & Palomar 48-in \\
\hline 55336.392 & $r$ & $>21.11$ & Palomar 48-in \\
\hline 55336.435 & $r$ & $>21.13$ & Palomar 48-in \\
\hline 55341.405 & $r$ & $>20.63$ & Palomar 48-in \\
\hline 55341.449 & $r$ & $>20.98$ & Palomar 48-in \\
\hline 55346.442 & $r$ & $>20.20$ & Palomar 48-in \\
\hline 55347.198 & $r$ & $>20.74$ & Palomar 48-in \\
\hline 55347.241 & $r$ & $21.23 \pm 0.29$ & Palomar 48-in \\
\hline 55352.204 & $r$ & $19.97 \pm 0.09$ & Palomar 48-in \\
\hline 55352.251 & $r$ & $20.04 \pm 0.10$ & Palomar 48-in \\
\hline 55357.232 & $r$ & $19.01 \pm 0.09$ & Palomar 48-in \\
\hline 55357.287 & $r$ & $19.06 \pm 0.08$ & Palomar 48-in \\
\hline 55362.238 & $r$ & $19.13 \pm 0.07$ & Palomar 48-in \\
\hline 55362.286 & $r$ & $19.16 \pm 0.06$ & Palomar 48-in \\
\hline 55367.269 & $r$ & $19.50 \pm 0.09$ & Palomar 48-in \\
\hline 55367.313 & $r$ & $19.49 \pm 0.10$ & Palomar 48-in \\
\hline 55372.395 & $r$ & $19.89 \pm 0.16$ & Palomar 48-in \\
\hline 55372.440 & $r$ & $19.94 \pm 0.16$ & Palomar 48-in \\
\hline 55377.394 & $r$ & $20.33 \pm 0.15$ & Palomar 48-in \\
\hline 55382.415 & $r$ & $21.13 \pm 0.25$ & Palomar 48-in \\
\hline 55382.460 & $r$ & $20.72 \pm 0.23$ & Palomar 48-in \\
\hline 55388.328 & $r$ & $21.15 \pm 0.24$ & Palomar 48-in \\
\hline 55389.182 & $r$ & $21.25 \pm 0.31$ & Palomar 48-in \\
\hline 55392.191 & $r$ & $21.23 \pm 0.15$ & Palomar 48-in \\
\hline 55395.188 & $r$ & $21.27 \pm 0.18$ & Palomar 48-in \\
\hline 55396.173 & $r$ & $21.48 \pm 0.25$ & Palomar 48-in \\
\hline 55400.174 & $r$ & $21.55 \pm 0.35$ & Palomar 48-in \\
\hline 55413.168 & $r$ & $>20.82$ & Palomar 48-in \\
\hline 55415.172 & $r$ & $>20.85$ & Palomar 48-in \\
\hline 55423.231 & $r$ & $>21.01$ & Palomar 48-in \\
\hline 55428.166 & $r$ & $>20.71$ & Palomar 48-in \\
\hline 55428.210 & $r$ & $>20.71$ & Palomar 48-in \\
\hline 55431.185 & $r$ & $>20.48$ & Palomar 48-in \\
\hline 55431.230 & $r$ & $>20.40$ & Palomar 48-in \\
\hline 55435.155 & $r$ & $>20.66$ & Palomar 48-in \\
\hline 55435.198 & $r$ & $>20.59$ & Palomar 48-in \\
\hline 55444.145 & $r$ & $>21.00$ & Palomar 48-in \\
\hline 55444.190 & $r$ & $>21.08$ & Palomar 48-in \\
\hline 55353.186 & $i$ & $19.65 \pm 0.13$ & Palomar 60-in \\
\hline 55353.188 & $r$ & $19.69 \pm 0.08$ & Palomar 60-in \\
\hline 55353.189 & $g$ & $19.88 \pm 0.11$ & Palomar 60-in \\
\hline 55353.305 & $i$ & $19.56 \pm 0.08$ & Palomar 60-in \\
\hline 55353.306 & $r$ & $19.56 \pm 0.22$ & Palomar 60-in \\
\hline 55353.308 & $g$ & $19.83 \pm 0.08$ & Palomar 60-in \\
\hline 55357.380 & $i$ & $19.07 \pm 0.09$ & Palomar 60-in \\
\hline 55357.382 & $r$ & $19.12 \pm 0.07$ & Palomar 60-in \\
\hline 55357.384 & $B$ & $19.75 \pm 0.41$ & Palomar 60-in \\
\hline 55357.387 & $g$ & $19.43 \pm 0.18$ & Palomar 60-in \\
\hline 55360.335 & $i$ & $18.90 \pm 0.10$ & Palomar 60-in \\
\hline 55360.337 & $r$ & $19.05 \pm 0.08$ & Palomar 60-in \\
\hline 55360.338 & $B$ & $20.14 \pm 0.25$ & Palomar 60-in \\
\hline 55360.340 & $z$ & $18.88 \pm 0.06$ & Palomar 60-in \\
\hline 55360.342 & $g$ & $19.73 \pm 0.10$ & Palomar 60-in \\
\hline 55362.379 & $i$ & $18.92 \pm 0.08$ & Palomar 60-in \\
\hline 55362.380 & $r$ & $19.17 \pm 0.10$ & Palomar 60-in \\
\hline 55362.382 & $B$ & $20.48 \pm 0.15$ & Palomar 60-in \\
\hline 55362.384 & $z$ & $18.92 \pm 0.08$ & Palomar 60-in \\
\hline 55362.386 & $g$ & $19.97 \pm 0.08$ & Palomar 60-in \\
\hline 55364.419 & $i$ & $18.95 \pm 0.08$ & Palomar 60-in \\
\hline 55364.420 & $r$ & $19.34 \pm 0.07$ & Palomar 60-in \\
\hline 55364.422 & $B$ & $20.82 \pm 0.23$ & Palomar 60-in \\
\hline 55364.432 & $g$ & $20.22 \pm 0.12$ & Palomar 60-in \\
\hline 55365.275 & $g$ & $20.30 \pm 0.07$ & Palomar 60-in \\
\hline
\end{tabular}

Table 3

(Continued)

\begin{tabular}{|c|c|c|c|}
\hline MJD & Filter & Mag & Facility \\
\hline 55366.461 & $i$ & $19.04 \pm 0.06$ & Palomar 60-in \\
\hline 55366.462 & $r$ & $19.47 \pm 0.09$ & Palomar 60-in \\
\hline 55366.464 & $B$ & $21.16 \pm 0.20$ & Palomar 60-in \\
\hline 55366.466 & $z$ & $18.71 \pm 0.11$ & Palomar 60-in \\
\hline 55367.294 & $g$ & $20.61 \pm 0.16$ & Palomar 60-in \\
\hline 55369.285 & $i$ & $19.21 \pm 0.07$ & Palomar 60-in \\
\hline 55369.286 & $r$ & $19.74 \pm 0.08$ & Palomar 60-in \\
\hline 55369.288 & $B$ & $>20.87$ & Palomar 60-in \\
\hline 55369.298 & $i$ & $19.28 \pm 0.07$ & Palomar 60-in \\
\hline 55369.302 & $r$ & $19.91 \pm 0.08$ & Palomar 60-in \\
\hline 55369.306 & $z$ & $18.93 \pm 0.09$ & Palomar 60-in \\
\hline 55370.301 & $g$ & $20.85 \pm 0.23$ & Palomar 60-in \\
\hline 55371.281 & $g$ & $21.14 \pm 0.22$ & Palomar 60-in \\
\hline 55372.263 & $i$ & $19.52 \pm 0.10$ & Palomar 60-in \\
\hline 55372.265 & $r$ & $20.08 \pm 0.12$ & Palomar 60-in \\
\hline 55372.269 & $z$ & $19.21 \pm 0.10$ & Palomar 60-in \\
\hline 55372.270 & $g$ & $21.12 \pm 0.28$ & Palomar 60-in \\
\hline 55376.268 & $i$ & $19.73 \pm 0.18$ & Palomar 60-in \\
\hline 55376.270 & $r$ & $20.46 \pm 0.10$ & Palomar 60-in \\
\hline 55376.284 & $z$ & $19.35 \pm 0.12$ & Palomar 60-in \\
\hline 55376.286 & $g$ & $>21.18$ & Palomar 60-in \\
\hline 55377.341 & $B$ & $>21.02$ & Palomar 60-in \\
\hline 55377.343 & $g$ & $>21.53$ & Palomar 60-in \\
\hline 55378.278 & $g$ & $21.64 \pm 0.26$ & Palomar 60-in \\
\hline 55379.276 & $i$ & $20.10 \pm 0.10$ & Palomar 60-in \\
\hline 55379.278 & $r$ & $20.77 \pm 0.11$ & Palomar 60-in \\
\hline 55379.286 & $z$ & $19.63 \pm 0.13$ & Palomar 60-in \\
\hline 55379.288 & $g$ & $>21.40$ & Palomar 60-in \\
\hline 55380.280 & $g$ & $>21.53$ & Palomar 60-in \\
\hline 55381.242 & $g$ & $>21.56$ & Palomar 60-in \\
\hline 55383.425 & $i$ & $20.19 \pm 0.10$ & Palomar 60-in \\
\hline 55383.427 & $r$ & $21.00 \pm 0.15$ & Palomar 60-in \\
\hline 55383.429 & $B$ & $>21.43$ & Palomar 60-in \\
\hline 55383.432 & $z$ & $19.69 \pm 0.14$ & Palomar 60-in \\
\hline 55383.440 & $g$ & $21.58 \pm 0.21$ & Palomar 60-in \\
\hline 55384.303 & $B$ & $>22.06$ & Palomar 60-in \\
\hline 55384.305 & $g$ & $22.42 \pm 0.19$ & Palomar 60-in \\
\hline 55386.422 & $i$ & $20.42 \pm 0.13$ & Palomar 60-in \\
\hline 55386.424 & $r$ & $21.14 \pm 0.14$ & Palomar 60-in \\
\hline 55386.430 & $z$ & $19.70 \pm 0.22$ & Palomar 60-in \\
\hline 55387.382 & $z$ & $19.78 \pm 0.19$ & Palomar 60-in \\
\hline 55434.288 & $i$ & $21.26 \pm 0.27$ & Palomar 60-in \\
\hline 55436.258 & $i$ & $21.78 \pm 0.26$ & Palomar 60-in \\
\hline 55436.264 & $r$ & $>21.86$ & Palomar 60-in \\
\hline 55442.230 & $i$ & $21.90 \pm 0.22$ & Palomar 60-in \\
\hline 55442.240 & $r$ & $22.61 \pm 0.27$ & Palomar 60-in \\
\hline 55443.237 & $i$ & $21.64 \pm 0.16$ & Palomar 60-in \\
\hline 55443.244 & $r$ & $>22.13$ & Palomar 60-in \\
\hline 55444.256 & $i$ & $21.71 \pm 0.19$ & Palomar 60-in \\
\hline 55447.244 & $i$ & $>21.16$ & Palomar 60-in \\
\hline 55444.263 & $r$ & $>21.44$ & Palomar 60-in \\
\hline 55461.206 & $i$ & $>20.98$ & Palomar 60-in \\
\hline 55478.158 & $i$ & $>21.39$ & Palomar 60-in \\
\hline 55479.139 & $i$ & $>21.33$ & Palomar 60-in \\
\hline 55479.146 & $r$ & $>21.88$ & Palomar 60-in \\
\hline 55485.126 & $i$ & $>21.45$ & Palomar 60-in \\
\hline 55477.168 & $g$ & $>22.20$ & Palomar 200-in \\
\hline 55477.178 & $r$ & $23.03 \pm 0.17$ & Palomar 200-in \\
\hline 55477.195 & $i$ & $21.97 \pm 0.09$ & Palomar 200-in \\
\hline 55486.119 & $r$ & $23.29 \pm 0.17$ & Palomar 200-in \\
\hline 55486.144 & $i$ & $22.02 \pm 0.20$ & Palomar 200-in \\
\hline 55486.168 & $g$ & $>21.65$ & Palomar 200-in \\
\hline 55593.655 & $r$ & $23.77 \pm 0.11$ & Keck I \\
\hline 55632.648 & $r$ & $23.73 \pm 0.06$ & Keck I \\
\hline
\end{tabular}


Table 3

(Continued)

\begin{tabular}{|c|c|c|c|}
\hline MJD & Filter & Mag & Facility \\
\hline \multicolumn{4}{|l|}{ PTF11bij } \\
\hline 55604.372 & $r$ & $>20.91$ & Palomar 48-in \\
\hline 55605.382 & $r$ & $>20.62$ & Palomar 48-in \\
\hline 55606.381 & $r$ & $>20.84$ & Palomar 48-in \\
\hline 55629.227 & $g$ & $20.48 \pm 0.18$ & Palomar 48-in \\
\hline 55629.270 & $g$ & $20.69 \pm 0.17$ & Palomar 48-in \\
\hline 55630.230 & $g$ & $20.57 \pm 0.18$ & Palomar 48-in \\
\hline 55631.238 & $g$ & $>20.95$ & Palomar 48-in \\
\hline 55632.213 & $g$ & $>20.62$ & Palomar 48-in \\
\hline 55633.187 & $r$ & $20.28 \pm 0.18$ & Palomar 48-in \\
\hline 55634.303 & $r$ & $20.49 \pm 0.18$ & Palomar 48-in \\
\hline 55634.347 & $r$ & $20.28 \pm 0.13$ & Palomar 48-in \\
\hline 55635.269 & $r$ & $20.12 \pm 0.16$ & Palomar 48-in \\
\hline 55635.312 & $r$ & $20.37 \pm 0.17$ & Palomar 48-in \\
\hline 55636.267 & $r$ & $>20.40$ & Palomar 48-in \\
\hline 55637.220 & $r$ & $>20.36$ & Palomar 48-in \\
\hline 55643.321 & $i$ & $20.42 \pm 0.13$ & Palomar 60-in \\
\hline 55643.323 & $r$ & $20.81 \pm 0.16$ & Palomar 60-in \\
\hline 55643.325 & $g$ & $21.38 \pm-99$ & Palomar 60-in \\
\hline 55649.167 & $i$ & $20.92 \pm 0.17$ & Palomar 60-in \\
\hline 55649.169 & $r$ & $>21.53$ & Palomar 60-in \\
\hline 55649.172 & $g$ & $>21.63$ & Palomar 60-in \\
\hline 55650.355 & $i$ & $21.26 \pm 0.14$ & Palomar 60-in \\
\hline 55650.362 & $r$ & $21.64 \pm 0.15$ & Palomar 60-in \\
\hline 55650.364 & $g$ & $>22.15$ & Palomar 60-in \\
\hline 55651.172 & $i$ & $>21.39$ & Palomar 60-in \\
\hline 55651.176 & $r$ & $>21.53$ & Palomar 60-in \\
\hline \multicolumn{4}{|l|}{ SN2007ke } \\
\hline 54345.51 & $R$ & $>17.69$ & KAIT \\
\hline 54355.48 & $R$ & $18.96 \pm 0.15$ & KAIT \\
\hline 54362.43 & $R$ & $18.26 \pm 0.13$ & KAIT \\
\hline 54367.42 & $R$ & $18.04 \pm 0.09$ & KAIT \\
\hline 54368.44 & $R$ & $17.99 \pm 0.07$ & KAIT \\
\hline 54369.38 & $R$ & $18.00 \pm 0.10$ & KAIT \\
\hline 54375.39 & $R$ & $18.22 \pm 0.13$ & KAIT \\
\hline 54380.48 & $R$ & $18.82 \pm 0.12$ & KAIT \\
\hline 54387.43 & $R$ & $>19.31$ & KAIT \\
\hline
\end{tabular}

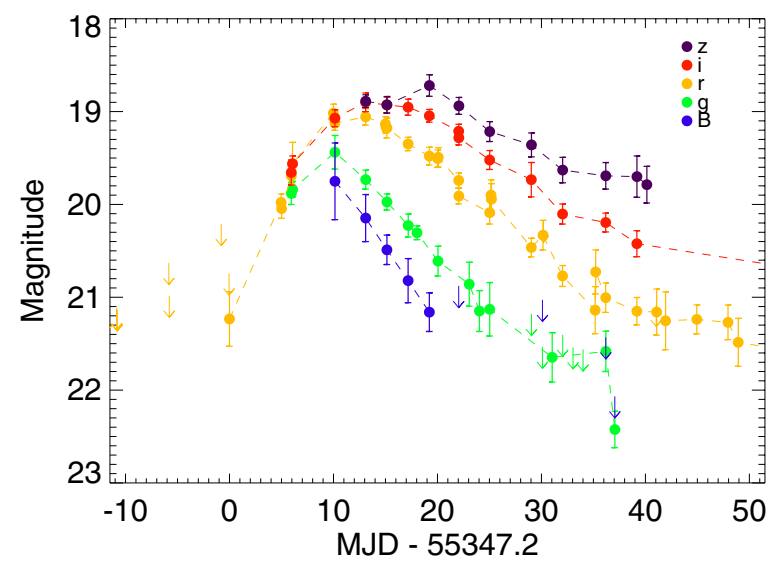

Figure 5. Light curves of PTF 10iuv. Note the rapid rise in $r$ band of 2 mag in 10 days, followed by the rapid decline at the rate of 1 mag in 12 days.

(A color version of this figure is available in the online journal.)

and Keck II (+DEIMOS) telescopes until the spectra became completely nebular (Figure 6).

The spectra evolved to show prominent helium features, resembling Type Ib spectra. The calcium lines become stronger

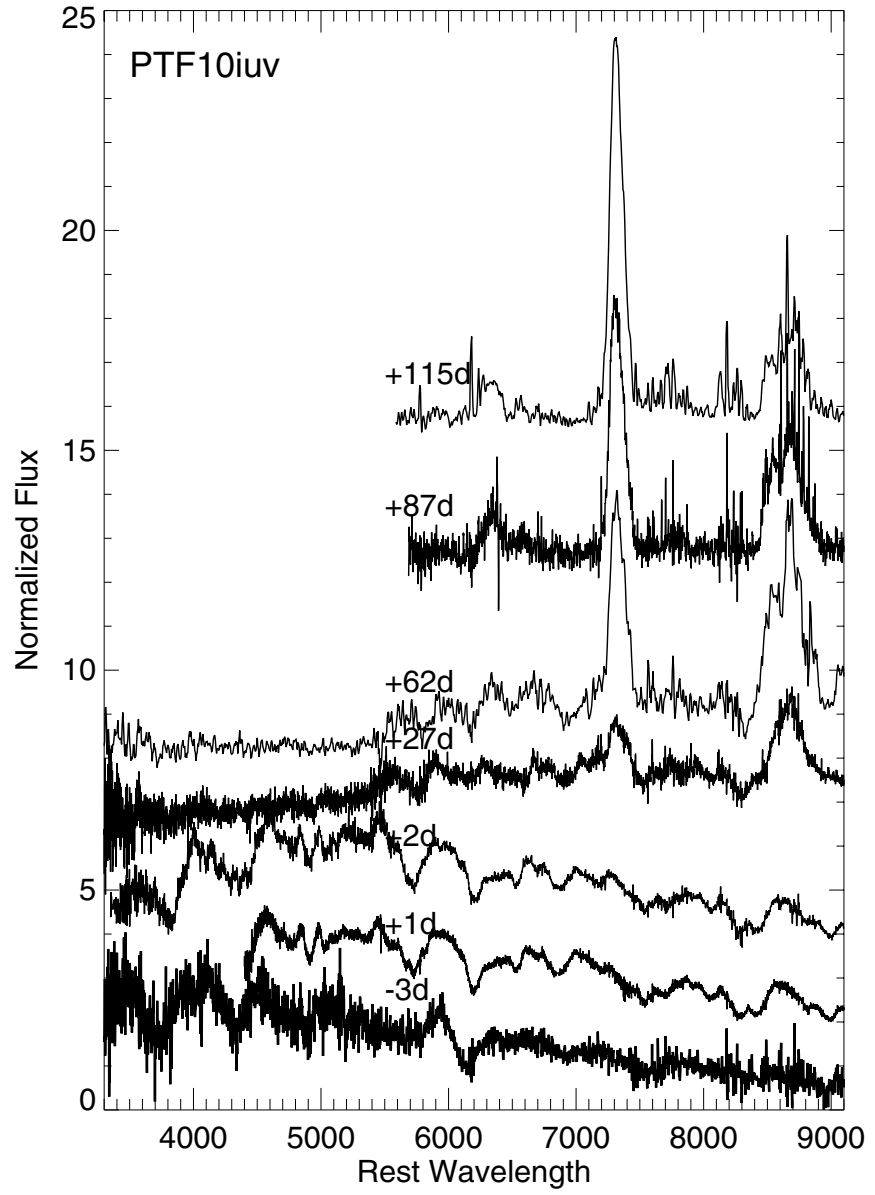

Figure 6. Spectral evolution of PTF 10iuv.

with time, especially [Ca II] relative to $\mathrm{O}$ I. We identify major lines both pre-maximum and post-maximum using SYNOW (Figure 7).

As with PTF 09dav, the spectrum of PTF 10iuv became nebular in only three months. Both showed prominent calcium emission (Table 2 and Figure 4). Unlike PTF 09dav, PTF 10iuv showed [O I] emission and did not exhibit $\mathrm{H} \alpha$ emission in the nebular phase. Note that subluminous Type II SN also show a lower ratio of oxygen to calcium relative to normal SN CC (Pastorello et al. 2004), but they also show prominent hydrogen emission.

\subsection{Radio Observations}

We observed PTF 10iuv with the Expanded Very Large Array twice, once on 2010 August 25.06 and again on 2011 May 12.20. We observed in the $X$ band $(8.46 \mathrm{GHz})$ and added together two adjacent $128 \mathrm{MHz}$ subbands with full polarization to maximize continuum sensitivity. Amplitude and bandpass calibration was achieved using the archival flux value of $\mathbf{J} 1721+3542$. Phase calibration was carried out every 10 minute by switching between the target field and the point source J1721+3542. The visibility data were calibrated and imaged in the AIPS package following standard practice.

The transient was not detected with a $3 \sigma$ upper limit of $189 \mu \mathrm{Jy}$ during the first epoch and $96 \mu \mathrm{Jy}$ during the second epoch. This corresponds to $L_{v}<1.0 \times 10^{27} \mathrm{erg} \mathrm{s}^{-1} \mathrm{~Hz}^{-1}$. 

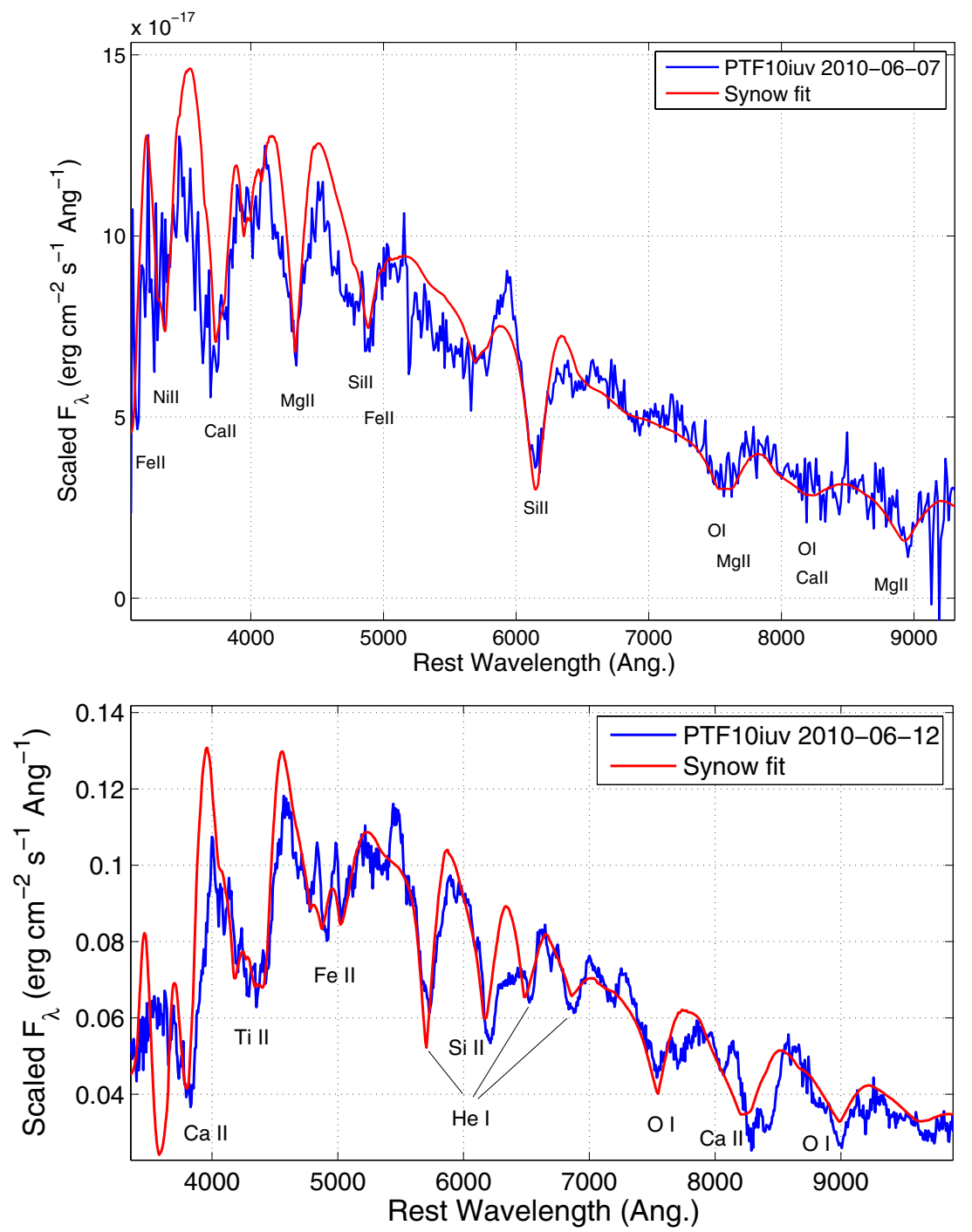

Figure 7. SYNOW fits to photospheric spectra of PTF 10 iuv. Top: SYNOW fit of a pre-maximum spectrum at phase of -3 days $\left(T \approx 8500 \mathrm{~K}\right.$ and $\left.v \approx 10,000 \mathrm{~km} \mathrm{~s} \mathrm{~s}^{-1}\right)$ with prominent magnesium, silicon, and oxygen lines. Bottom: SYNOW fit of post-maximum photospheric spectrum at phase of +2 days of PTF $10 \mathrm{iuv}(T \approx 6500 \mathrm{~K}$ and $v \approx 9000 \mathrm{~km} \mathrm{~s}^{-1}$ ). The most prominent features are helium, oxygen, calcium, and titanium.

(A color version of this figure is available in the online journal.)

\section{OBSERVATIONS: PTF 11BIJ}

On 2011 March 9.227, PTF discovered a new transient, PTF $11 \mathrm{bij}$, at $\alpha(\mathrm{J} 2000)=12^{\mathrm{h}} 58^{\mathrm{m}} 58^{\mathrm{s}} .39$ and $\delta(\mathrm{J} 2000)=$ $+37^{\circ} 23^{\prime} 12^{\prime \prime}$. 0 . It was initially found at $g=20.5 \mathrm{mag}$, and we monitored its brightness with the P60 in the gri filters. Similar to PTF 09dav and PTF 10iuv, the light curve showed a maximum observed luminosity in the gap between novae and supernovae, and it evolved rapidly (see Figure 8 and Table 3).

We obtained a target of opportunity spectrum on 2011 April 3.40 and a nebular spectrum on April 27.40, both with LRIS on the Keck I telescope (Figure 4). The spectra were calcium-rich, similar to PTF 10iuv and PTF 09dav, and the redshift of 0.035 is consistent with a group of early-type galaxies (the nearest galaxy is offset by $33 \mathrm{kpc}$ ). The nebular spectra also show some [O I] emission similar to PTF 10iuv.

\section{OBSERVATIONS: ARCHIVAL CANDIDATES}

Here, we present archival data on candidate transients from other surveys noted for their calcium-rich spectra (Filippenko et al. 2003). Specifically, Perets et al. (2010) sug-



Figure 8. Light curves of five members of the class of calcium-rich gap transients. Note the lower peak luminosities and faster evolution relative to normal supernovae.

(A color version of this figure is available in the online journal.) 




Figure 9. Locations of calcium-rich supernovae from the KAIT Lick Observatory Supernova Search sample. From top left to bottom right: SN 2000ds, SN 2003H, SN 2003dg, SN 2003dr, SN 2005E, and SN 2007ke. Unfortunately, adequate photometric data are available only for SN 2005E and SN 2007ke to confirm their similarity to PTF 09dav, PTF 10iuv, and PTF 11bij. Note that some of the locations are atypical: elliptical host, off the disk of an edge-on galaxy, large projected offset from isolated host, and galaxy group environment.

gest that the following six supernovae resemble SN 2005E: SN 2000ds (IAUC\#7507), SN 2001co (IAUC\#7643), SN 2003dg (IAUC\#8113), SN 2003dr (IAUC\#8117), SN 2003H (IAUC\#8045), and SN 2007ke (CBET\#1084). SN 2000ds was discovered by T. Puckett. SN 2001co, SN 2003dg, SN 2003H, and SN 2007ke were discovered by LOSS. SN 2003dr was discovered independently by both T. Puckett and LOSS.

Here, we review the available data for these supernovae. In Figure 9, we display the environments of these candidate members of the calcium-rich class. Next, in Figure 10, we plot their spectra (at the latest phase available) and compare them to those of PTF 10iuv. The strength of [Ca II] relative to oxygen is a distinguishing spectroscopic feature of this class; it is common to SN 2000ds, SN 2003dg, SN 2003dr, SN 2007ke, and SN 2003H. The case for SN 2001co is less clear due to the noisy spectrum and we exclude it from further analysis.

Unfortunately, the photometry is extremely sparse for this sample - only one of the five supernovae has more than two points on the light curve. SN 2007ke has a peak absolute magnitude of -16.3 , a rise time of 15 days, and a decline rate of $0.1 \mathrm{mag} \mathrm{day}^{-1}$ (Figure 8 and Table 3). Therefore, SN 2007ke satisfies all of the photometric and spectroscopic properties 
Table 4

Summary of Properties of "Ca-rich Gap" Transients

\begin{tabular}{lccccc}
\hline \hline & SN 2005E & SN 2007ke & PTF 09dav & PTF 10iuv & PTF 11bij \\
\hline Peak absolute mag. $\left(M_{r}\right)$ & -15.5 & -16.3 & -16.4 & -16 & -15.9 \\
Rise time (days) & $>9$ & 15 & 12 & 12 & $\ldots$ \\
Decay time (days) & 14 & 15 & 12 & 12 & 10 \\
Photospheric velocity $\left(\mathrm{km} \mathrm{s}^{-1}\right)$ & 11,000 & 11,000 & 6000 & 10,000 & $\ldots$ \\
Helium in photospheric spectra? & Yes & Yes & Maybe & Yes & $\ldots$ \\
Calcium in nebular spectra? & Yes & Yes & Yes & Yes & Yes \\
Hydrogen in nebular spectra? & No & No & No & No & Yes \\
Oxygen in nebular spectra? & Yes & Maybe & No & -12.1 & -12.4 \\
Limiting magnitude of host galaxy & -7.5 & $\ldots$ & 40 & 37 & 33 \\
Offset from nearest host galaxy $(\mathrm{kpc})$ & 23 & 8.5 & No & Yes & Yes \\
Galaxy group environment? & No & Yes & & & \\
\hline
\end{tabular}

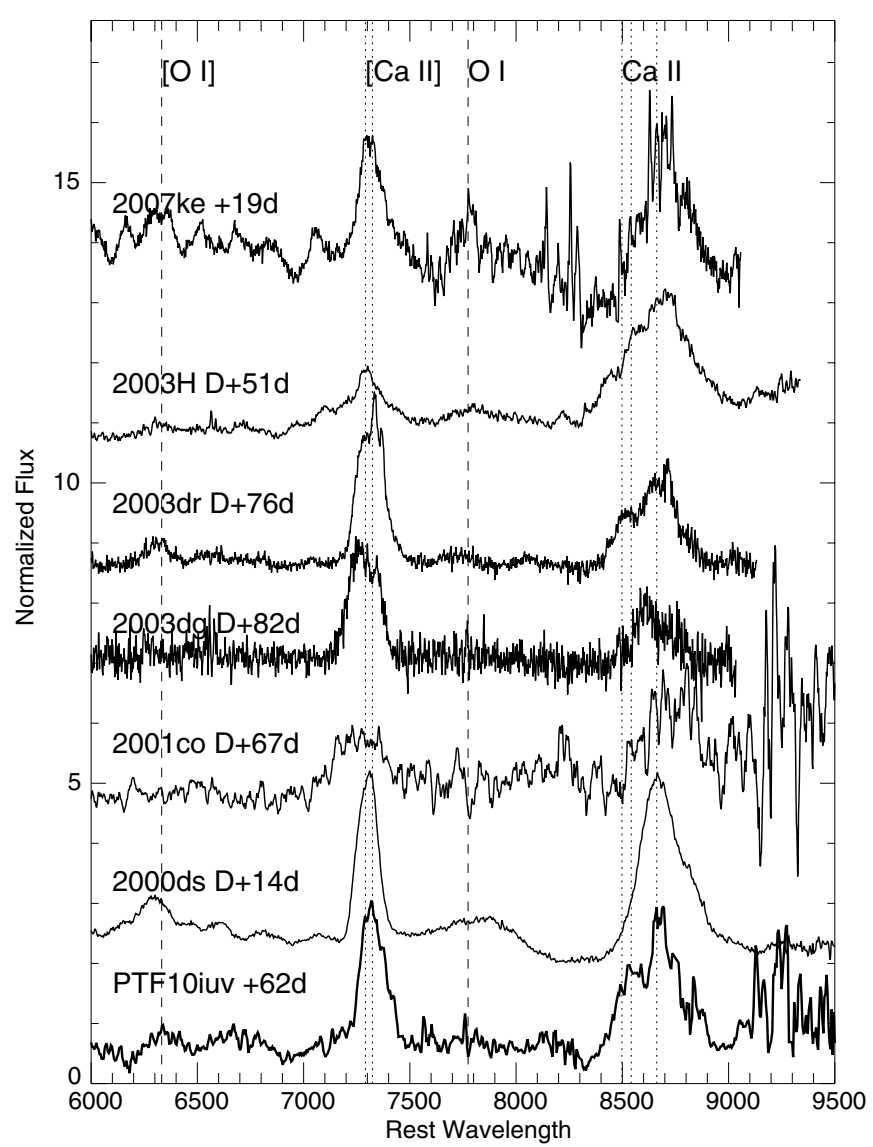

Figure 10. Spectra of the KAIT/LOSS sample of calcium-rich supernovae compared to PTF 10iuv. Except for SN 2007ke, as the photometry is not available to constrain the maximum-light epoch, the phase is relative to discovery and not maximum.

of the class of "calcium-rich gap" transients as outlined in Section 1. Note that the location of SN 2007ke is "off-galaxy" (Figure 9 (bottom-right panel)). In view of the paucity of photometric data for the other four supernovae, we do not discuss them further.

\section{ANALYSIS}

\subsection{The Sample}

To summarize, the five distinguishing characteristics of our peculiar new class of transients are (1) peak luminosity intermediate between that of novae and most supernovae, (2) faster photometric evolution (rise and decline) than that of normal supernovae, (3) photospheric velocities comparable to those of normal supernovae, (4) early evolution to the nebular phase, and (5) nebular spectra dominated by Calcium emission.

Based on available observations, the objects that satisfy the defining characteristics of this class of "calcium-rich gap" transients are SN 2005E, SN 2007ke, PTF 09dav, PTF 10iuv, and PTF 11bij. Their light curves are shown in Figure 8 and nebular spectra in Figure 4. Observed properties of these events are summarized in Table 4.

Our choice of these five characteristics is motivated by choosing the maximum set of characteristics that gives the largest number of related transients. Evidently, if we add characteristics to this list, the family size becomes smaller and we may overlook a unifying explanation. If we remove characteristics from this list, the family becomes larger but we may inadvertently introduce a diversity of progenitor channels. We discuss examples of each case below.

If we require an additional sixth property of a photospheric spectrum with prominent helium lines, only PTF 10iuv and SN 2005E would be members of the family. It is not clear whether the photospheric spectra of PTF 09dav exhibit helium (see discussion in Sullivan et al. 2011). The earliest spectra of SN 2007ke and PTF 11 bij are not until +19 day and +21 day after maximum light. These two spectra already show the nebular features of prominent [Ca II] emission common to the class, but we cannot confirm helium. Therefore, we allow a photospheric diversity but require a nebular uniformity in the class.

If in addition to calcium, we also require oxygen emission in the nebular spectrum, only PTF 10iuv, SN 2005E, and PTF 11 bij would be members of this class. PTF 09dav does not show oxygen emission and SN 2007ke does not have spectra at a sufficiently late epoch to test this.

On the other hand, if we require the absence of hydrogen emission from this class, we would exclude PTF 09dav. It is the only member of this class that showed hydrogen, albeit only at late times.

It is pertinent here to discuss the case of SN 2008ha (Valenti et al. 2009; Foley et al. 2009, 2010), a peculiar transient found in an irregular galaxy. This transient satisfies four out of the five properties of this class. The only difference is that it showed extremely low photospheric velocities of $\approx 2000 \mathrm{~km} \mathrm{~s}^{-1}$. The photospheric spectra of SN 2008ha have been compared to those of the SN 2002cx family of supernovae, and it has been suggested that SN 2008ha is a lower velocity and lower luminosity analog of this family. The SN 2002cx family has starkly different late-time spectra dominated by permitted iron lines (it has been argued that these spectra are not nebular even at +400 day, e.g., Jha et al. 2006; Sahu et al. 2008) and no 
evidence of calcium-richness. Therefore, the 2002cx family is likely unrelated to the class of transients discussed here.

The membership of SN 2008ha in either the SN 2002cx family or in the "calcium-rich gap" class of transients is unclear. On grounds that the extremely low velocities may be diagnostic of a different explosion mechanism, we tentatively exclude SN 2008ha as a member of the class of "calcium-rich gap" transients.

Originally, Filippenko et al. (2003) suggested that there was a class defined by only one property: prominence of calcium relative to supernovae of Type Ib. This property alone would suggest that SN 2000ds, SN 2003dg, SN 2003dr, and SN 2003H are also members of this class. As discussed in Section 6, these events were not monitored photometrically. Additionally, SN 2005cz (Kawabata et al. 2010; Perets et al. 2011), which also has a similar nebular spectrum but an extremely sparse light curve, may also become a member of this class (albeit the broad $\mathrm{H} \alpha$ emission; Kawabata et al. 2010). Another possible member of this class is a supernova offset from the galaxy UGC11021. It was discovered by MASTER (ATEL \#3610) and showed some evidence of calcium-richness (ATEL \#3615); neither a light curve nor a nebular spectrum are available for this transient.

Without a light curve, the ejecta mass in this classification would remain ambiguous. As discussed below, low ejecta mass is a crucial clue to distinguish this class from variants of normal $\mathrm{SNe} \mathrm{CC}$ as well as $\mathrm{SNe}$ Ia. We note here that even if light curves were available and consistent with the properties of this class, it would not alleviate the challenge posed to many progenitor scenarios by the strong preference for remote locations. Specifically, SN 2005cz is in an old environment (Perets et al. 2011), SN 2000ds is in an elliptical galaxy, and SN 2003dr is offset from an edge-on galaxy.

\subsection{Comparison to Type Ia and Type Ib Supernovae}

In Sullivan et al. (2011), we discussed that although the photospheric spectra of PTF 09dav share some similarities to those of SNe Ia, there are several differences as follows. (1) PTF 09dav does not obey the Phillips relation (Phillips 1993). There is a break in the slope of the relation for subluminous SN Ia, but this is obeyed by all SN 1991bg-like supernovae including the least luminous member, SN 2007ax (Kasliwal et al. 2008). (2) The photospheric spectra of PTF 09dav show scandium and strontium (elements usually seen only in spectra of SN CC). (3) The nebular spectrum of PTF 09dav does not show any Fe-peak elements, which is inconsistent with nebular spectra of all SN Ia (Figure 4).

The photospheric spectra of PTF 10iuv around maximum light resemble those of $\mathrm{SNe} \mathrm{Ib}$. However, a distinguishing characteristic is that a month after maximum, calcium emission starts to become prominent. We checked spectra of $26 \mathrm{SNe} \mathrm{Ib}$ from PTF for calcium-richness. In Figure 11, we show seven spectra of SNe Ib. While the calcium near-IR triplet becomes prominent in all of the $\mathrm{SN}$ Ib as they evolve, the [Ca II] doublet is especially prominent in PTF 10iuv. PTF 10vnv and PTF 10inj also show $[\mathrm{Ca} \mathrm{II}]$, but the relative flux ratio of $\left[\mathrm{Ca}\right.$ II] to $\mathrm{O}_{\mathrm{I}}$ is much lower than that seen in PTF 10iuv. The case of PTF 10hcw is less clear, as [Ca II] is more prominent than $\mathrm{O}_{\mathrm{I}}$ but the supernova set soon after discovery and the photometric coverage stops before it reached maximum. In the nebular phase, the flux ratio of $[\mathrm{Ca}$ II] to $[\mathrm{O} \mathrm{I}]$ is also much lower in $\mathrm{SNe}$ Ib relative to PTF 10iuv (Figure 4). Furthermore, the light curves of SNe Ib

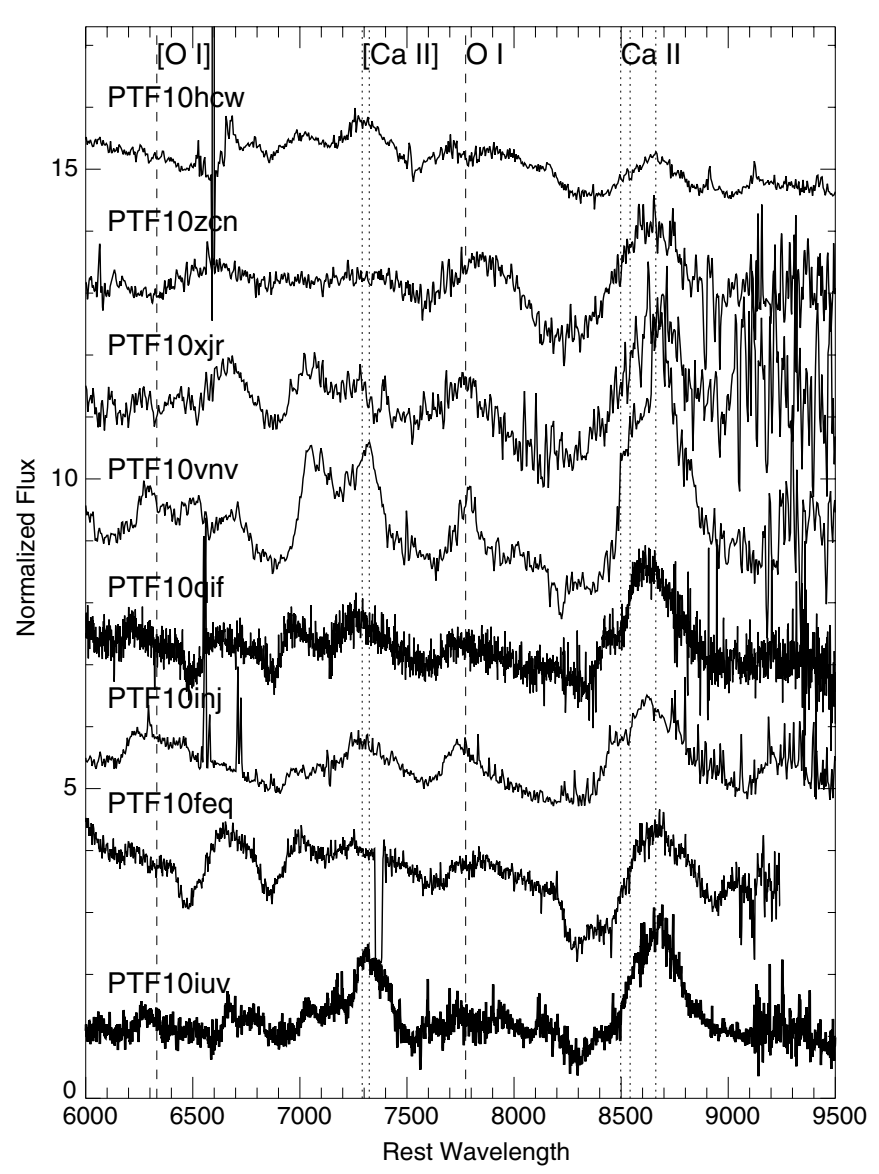

Figure 11. Comparison of PTF 10iuv to other SN Ib about a month after maximum light. Note the strength of the $[\mathrm{Ca} \mathrm{II}]$ line relative to $\mathrm{O}$ I.

are typically more luminous by about $2 \mathrm{mag}$ and evolve more slowly by at least a factor of two.

\subsection{Modeling the Light Curve: Ejecta Mass and Radioactivity}

The light curve of PTF 10iuv is very well sampled. The rising portion of the light curve can constrain the ejecta mass and the late-time decay can constrain the radioactive mass. Therefore, we can test the hypothesis of whether this explosion is radioactively powered by ${ }^{56} \mathrm{Ni}$ as in $\mathrm{SN}$ Ia.

For supernovae with photospheric velocity, $v$, and rise time, $t_{\mathrm{r}}$, the ejecta mass is $M_{\mathrm{ej}} \propto v t_{\mathrm{r}}^{2}$ (Arnett 1982). Therefore, assuming the same opacity, we can derive an ejecta mass by scaling relative to a normal SN Ia with parameters $1.4 M_{\odot}, 11,000 \mathrm{~km} \mathrm{~s}^{-1}$, and 17.4 days. PTF 10iuv has a rise time of 12 days and an average photospheric velocity of $7600 \mathrm{~km} \mathrm{~s}^{-1}$; hence, it has an ejecta mass of $0.46 M_{\odot}$. PTF 09dav has the same rise time but a lower velocity of $6000 \mathrm{~km} \mathrm{~s}^{-1}$, giving an ejecta mass of $0.36 M_{\odot}$ (Sullivan et al. 2011). There are no data to constrain the rise time of SN 2005E. Assuming it was also 12 days and using its photospheric velocity of $11,000 \mathrm{~km} \mathrm{~s}^{-1}$, we get an ejecta mass of $0.67 M_{\odot}$. Note that this is a factor of two higher than the total ejecta mass estimated by Perets et al. (2010) based on the nebular spectrum. This may not be surprising given that the nebular spectrum analysis may not have accounted for all of the helium. Moreover, higher ejecta mass for SN 2005E was expected based on light-curve modeling as well (Waldman et al. 2011).

For PTF 10iuv, given the peak luminosity of $4.6 \times$ $10^{41} \mathrm{erg} \mathrm{s}^{-1}$ and a 12 day rise, we can derive a ${ }^{56} \mathrm{Ni}$ mass 


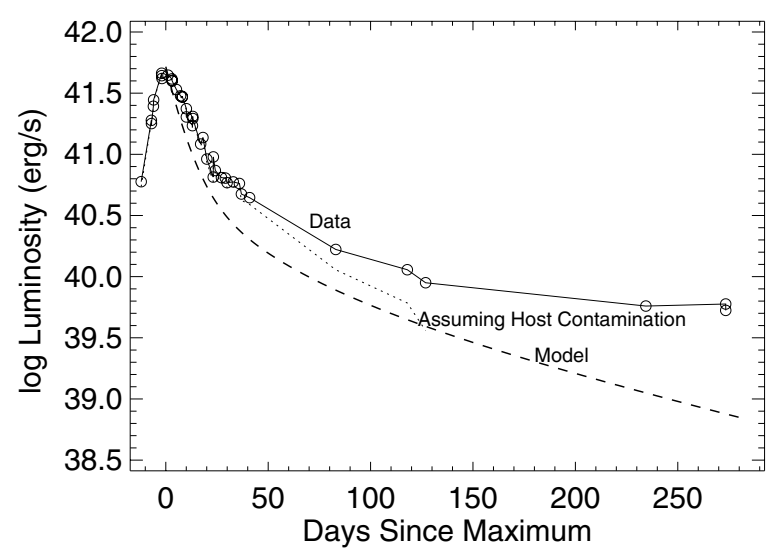

Figure 12. Modeling the light curve (circles; $v F_{v}$ in the $r$ band) of PTF 10iuv with radioactive ${ }^{56} \mathrm{Ni}$ decay. The dotted line assumes that the last two data points are due to host-galaxy light contamination and subtracts $r=23.8$ mag from the rest of the light curve. The model (dashed line) is based on an ejecta mass of $0.46 M_{\odot}$ and an ${ }^{56} \mathrm{Ni}$ mass of $0.016 M_{\odot}$.

of $0.016 M_{\odot}$. However, we find that the late-time photometry is brighter than what is expected from radioactive decay of ${ }^{56} \mathrm{Ni}$ (Figure 12). A possible caveat here is that there is some light from an underlying dwarf host galaxy. The last photometric point is fainter than the depth of our pre-explosion co-add. It is possible that there is some contamination in the light curve due to the flux of such a host. Therefore, we plot the dotted line in Figure 12, which subtracts the last two data points from the rest of the light curve. We are continuing to obtain even later time imaging to further test this caveat.

Should further observations rule out host contamination, this may suggest either a radioactive species other than ${ }^{56} \mathrm{Ni}$ or an additional source powering the light curve. For example, a larger contribution by ${ }^{44} \mathrm{Ti}$, which has a long half-life of $60 \mathrm{yr}$, may seem a promising candidate to explain the late-time slow evolution. However, if all of the high luminosity at late times were attributed to this element, it would require too large a quantity of ${ }^{44} \mathrm{Ti}\left(\sim 2 M_{\odot}\right)$. Modeling of different combinations of other additional radioactive species $\left({ }^{44} \mathrm{Cr},{ }^{44} \mathrm{Ti}\right.$, and $\left.{ }^{52} \mathrm{Fe}\right)$ in the context of helium-shell detonations on small (Waldman et al. 2011) and large CO white dwarf cores (Shen et al. 2010) has been undertaken. These efforts were limited by the missing light-curve data in the rising and very late phase of SN 2005E. The well-sampled light curve of PTF 10iuv should be able to better constrain these models.

\subsection{Constraint on Electron Density}

Using the flux ratio between [Ca II] and the Ca II near-IR triplet, we can constrain the density given a temperature. For PTF 10iuv, the nebular spectrum at +87 day gives a ratio of 0.86 and the spectrum at +115 day gives a ratio of 1.8 . For PTF 09dav, the nebular spectrum at +94 day gives a ratio of $>2.2$ (assumed width of $300 \AA$, $3 \sigma$ limit is $3 \times 10^{-18} \mathrm{erg} \mathrm{cm}^{-2} \mathrm{~s}^{-1} \AA^{-1}$ ). For SN 2005E, the ratio at +65 day is 0.55 (Perets et al. 2010). SYNOW fits to late-time spectra suggest a temperature roughly in the range 4500-5000 K. Following Figure 2 of Ferland \& Persson (1989), assuming a temperature around $4500 \mathrm{~K}$, the electron density is on the order of $10^{9} \mathrm{~cm}^{-3}$ and decreases by a factor of a few in a couple of months.

\subsection{Modeling the Nebular Spectra}

Next, we estimate the mass of the dominant species in the ejecta using the nebular spectrum.
We estimate the oxygen mass based on the luminosity of the [O I] line in the nebular phase. We can assume the high-density limit holds $\left(>10^{6} \mathrm{~cm}^{-3}\right)$ and estimate the oxygen mass as

$$
M_{O}=10^{8} f_{[\mathrm{O} \mathrm{I}]} D_{\mathrm{Mpc}}^{2} e^{\left(2.28 / T_{4}\right)} M_{\odot},
$$

following Uomoto (1986). Assuming a temperature of $4500 \mathrm{~K}$, we get $0.025 M_{\odot}$ of oxygen for PTF $10 \mathrm{iuv}$ and $0.037 M_{\odot}$ for SN 2005E. Note that the oxygen mass is consistent with the mass derived by Perets et al. (2010). A cautionary note here is that this calculation is extremely sensitive to temperature; a difference of only $500 \mathrm{~K}$ in temperature changes this estimate by a factor of two.

Two months after maximum brightness, the luminosity in [Ca II] nebular emission for SN 2005E was $2 \times 10^{39} \mathrm{erg} \mathrm{s}^{-1}$ and the derived ejecta mass was $0.135 M_{\odot}$ (Perets et al. 2010). The [Ca II] nebular luminosity is a factor of 2.5 smaller for PTF 10iuv and factor of 2.6 larger for PTF 09dav relative to SN 2005E. Under similar conditions, this may be representative of the range in calcium mass for these events.

\section{DISCUSSION}

We are struck by the remote "off-galaxy" locations of all five confirmed members of the class of "calcium-rich gap" transients (Figures 3 and 9). While five may still be considered smallnumber statistics, location can be a powerful diagnostic of the physical origin. Below, we discuss the pros and cons of two broad classes of possible progenitor scenarios: a white dwarf and a massive star.

\subsection{A White Dwarf?}

The white dwarf scenario has tremendous appeal to explain "calcium-rich gap" transients for two reasons. First, the remote galactic locations, intracluster environments, and constraints on star formation of the observed members of this family point to an old population. Second, the low ejecta masses derived from the light curves and nebular spectra are in the range of what is normally derived from white dwarf explosions and not massive star explosions.

The family of transients presented here is not the standard thermonuclear explosion of a white dwarf as seen in SNe Ia. There are two major differences. First, none of these transients obey the Phillips (1993) relation. The inferred ejected masses are smaller than in SNe Ia. Second, Fe-group elements seen in all SNe Ia are absent in the nebular spectra of these explosions.

Next, we discuss several alternate explosions of white dwarfs that have been discussed in the literature to explain intermediateluminosity and fast-evolving transients. First, we consider a "Ia" explosion following the final helium flash in an ultracompact white-dwarf-white-dwarf binary (Bildsten et al. 2007; Shen et al. 2010). The observed rise time of "calcium-rich gap" transients is too slow for a ".Ia" explosion. The observed ejecta masses are too large for a shell detonation (Waldman et al. 2011) and suggest that the core also participated in the explosion.

Another possibility is deflagration of a sub-Chandrashekharmass white dwarf (Woosley \& Weaver 1994), as it is also expected to have lower luminosity than an SN Ia. However, the late-time light curve and absence of Fe-peak elements in the nebular spectra are inconsistent with this model.

Woosley \& Kasen (2011) derive several models for explosions of sub-Chandrashekhar white dwarfs. However, the peak absolute magnitude and rise-time range observed for "calciumrich gap" transients are not predicted by any of these models. 
Another proposed theoretical model is accretion-induced collapse (AIC) of a rapidly rotating white dwarf into a neutron star (Metzger et al. 2009). However, AIC predicts a spectrum dominated by intermediate-mass elements, much higher velocities $(0.1 c)$, and much more rapid rise (one day) and decline (four to five days) than what is observed for Ca-rich gap transients.

The presence of helium in SN 2005E and PTF 10iuv can be explained for some white dwarf models, but the presence of hydrogen in PTF 09dav poses a major challenge to all white dwarf explosions. One possible scenario to explain late-time hydrogen in a white dwarf explosion is as follows: consider a binary where mass transfer is from a hydrogen-rich companion star onto a white dwarf. This accretion initially proceeded at a low rate, resulting in a series of nova eruptions prior to the sub-Chandrashekhar explosion or helium-shell detonation. The photons from the final explosion would eventually reach one of the previously ejected nova shells and the interaction would give $\mathrm{H} \alpha$ emission.

Quantitatively, if the shell was photoionized, the distance to this nova shell would be the speed of light multiplied by 95 days, or $2.5 \times 10^{17} \mathrm{~cm}$. Given the velocity of $1000 \mathrm{~km} \mathrm{~s}^{-1}$ to traverse this distance, the nova eruption would then have occurred $78 \mathrm{yr}$ prior to the supernova. Given the absence of hydrogen in earlytime spectra, we consider instead collisional ionization when the shock front itself reaches the shell. The distance to the shell would then be $8.2 \times 10^{15} \mathrm{~cm}$ and the nova shell would have to be ejected $\sim 950$ days before the supernova. The mass of hydrogen needed to sustain the observed luminosity for an hour is $4 / n \times 10^{-4} M_{\odot}$, where $n$ is the number of times the same hydrogen atom gets excited in one hour.

Yet another scenario was recently proposed by Metzger (2012) that can potentially explain a trace amount of hydrogen-tidal disruption of a white dwarf by a neutron star or stellar mass black hole. However, several other observables (e.g., velocities) are inconsistent with this model.

While the presence of five transients in remote locations is a strong clue in favor of the white dwarf scenario, the absence of any events in disks of galaxies is quite problematic. Studies of white dwarf binaries have shown that the number of white dwarfs in halos relative to that in the disk is nearly a third, larger than expected from the ratio of the overall mass or light (Ruiter et al. 2007). However, that is not sufficient to explain why there is a strong preference for remote locations.

If the progenitor is indeed a white dwarf, then there must be a characteristic of white dwarfs which can be attributed to location and affects the explosion signature. For example, the age of the white dwarf (e.g., if the timescale for evolution in a multiple star system were longer), the composition of the white dwarf (e.g., if helium white dwarfs were preferentially found in remote locations), and environmental characteristics (e.g., if metallicity/density affected the explosion or progenitor evolution timescale). One scenario that remains to be tested with detailed modeling is that at lower metallicity, even relatively lower mass stars can evolve to become white dwarfs in a Hubble time. Perhaps, explosions of these relatively lower mass white dwarfs are subluminous, fast evolving, and calcium-rich.

\subsection{A Massive Star?}

Given that none of the above white dwarf models easily explain all of the observed clues of "calcium-rich gap" transients, we briefly consider the massive star option. There are some explosion properties which are reminiscent of SNe CC: similarity of nebular spectra albeit with enhanced calcium, similarity of photospheric spectra to $\mathrm{SNe} \mathrm{Ib}$ in the case of PTF 10iuv and SN 2005E, and the presence of hydrogen in the case of PTF 09dav.

\subsubsection{Massive Star in the Outskirts?}

Perets et al. (2010) convincingly argue against the progenitors being hypervelocity massive stars formed in the disk. A more feasible option that allows massive stars to traverse large distances is tidal stripping during galaxy interactions. This is especially intriguing given the galaxy group surrounding the locations of PTF 10iuv, PTF 11bij, and SN 2007ke.

Studies of intracluster environments show evidence for a dominant old component as well as a young component, particularly in tidal tails of interacting gas-rich galaxies (Mullan et al. 2011; Williams et al. 2007). It has been shown that roughly $15 \%$ of the cluster's mass is in the intracluster medium. Sivanandam et al. (2009) show that $50 \%$ of metals come from intracluster supernovae. Mullan et al. (2011) present a study of tidal tails of interacting galaxies and find that young star clusters formed in situ in half of the sample. Another possibility is that there is some low-level star formation in remote locations. Recent systematic surveys (Werk et al. 2008, 2010) show star formation in the far outskirts of noninteracting galaxies. About $10 \%$ of the galaxies in this study had outlying $\mathrm{H}$ II regions with offsets between $20 \mathrm{kpc}$ and $40 \mathrm{kpc}$.

Our deep ground-based limits show neither any evidence of tidal tails nor $\mathrm{H}$ II regions. However, given the distance to these supernovae, they are not sensitive to a significant fraction of the luminosity function of $\mathrm{H}$ II regions and young star clusters. So, we cannot completely rule out the possibility of a massive star origin. Deep narrowband imaging will provide better constraints.

\subsubsection{Fallback Supernova?}

Assuming there was a small amount of in situ star formation below our detection limits, one would expect regular SN CC in the outskirts as well. However, the absence of regular SN CC with offsets larger than $30 \mathrm{kpc}$ in the PTF sample appears to be at odds with the progenitors being massive stars (see Figure 1).

A possible partial resolution is if the fate of massive stars in very low metallicity environments is very different. Specifically, the lower metallicity in the outskirts lowers the mass-loss rate, and it has been suggested that a larger fraction of massive stars collapse directly to a black hole (e.g., Heger et al. 2003; O'Connor \& Ott 2011). Such a collapse results in a subluminous explosion or no explosion at all. This could explain both the small numbers of SNe CC in the outskirts and the dearth of regular $\mathrm{SNe} \mathrm{Ib} / \mathrm{c}$ in low-metallicity environments. This is also consistent with studies that show that regular $\mathrm{SNe} \mathrm{Ib} / \mathrm{c}$ are more centrally concentrated relative to SNe II (Anderson \& James 2009). Some of the missing Type Ib/c explosions in lowmetallicity environments (outer parts of galaxies) could perhaps be subluminous and short-lived fallback events. Fallback of some ejecta onto the proto-neutron star to form the black hole could explain the low ejecta mass, fast evolution, and absence of heavy elements observed in this class. It would not be surprising, then, that the first fallback events observed are located in the outskirts of their hosts.

There are three important caveats to this scenario. First, since the initial mass function is quite universal and varies little over a wide range of metallicity (e.g., Myers et al. 2011), we expect to see a significant population of normal SNe II-P from the lower mass range of SNe CC unaffected by metallicity. But, 
current transient surveys have not found any SNe II-P in such remote galactic outskirts. Second, PTF 10iuv and SN 2005E were hydrogen-free. Therefore, mass loss would have to be finely tuned to be high enough to expel the hydrogen, low enough such that there is fallback onto the core, yet not too low such that there is no explosion at all. Kawabata et al. (2010) proposed an alternate scenario where the hydrogen was removed by close binary interaction. Third, some galaxies have shown that the metallicity gradient flattens beyond a certain radius and does not continue to decrease. For example, metallicity measurements of the $\mathrm{H}$ II regions in the outskirts show that they are not exceptionally low metallicity (roughly 0.4 solar; Werk et al. 2011). To the extent that metallicity is diagnostic of linedriven mass loss, this presents another challenge to the massive star scenario.

\section{CONCLUSIONS}

Five transients (SN 2005E, SN 2007ke, PTF 09dav, PTF 10iuv, and PTF 11bij) share the following common properties: low peak luminosity, fast photometric evolution, large photospheric velocities, early evolution to the nebular phase, and calcium-dominated ejecta. Furthermore, all five members of this class of "calcium-rich gap" transients are located in the outskirts of their putative host galaxies. This set of properties, in conjunction with peculiarities specific to each of them (e.g., the presence of hydrogen, scandium, and strontium in PTF 09dav, strong constraints against star formation in SN 2005E, intracluster environment of PTF 10iuv, PTF 11bij, and SN 2007ke), warrants a creative modification of standard thermonuclear or standard core-collapse scenarios.

We can estimate a lower limit on the rate of this class of "calcium-rich gap" events by comparing to the rate of SNe Ia discovered by PTF in the same volume in the same time. Within $200 \mathrm{Mpc}$, we found $128 \mathrm{SN}$ Ia and 3 such events. Therefore, a lower limit on the rate is $>7 \times 10^{-7} \mathrm{Mpc}^{-3} \mathrm{yr}^{-1}$ (we used the SN Ia rate from Li et al. 2011). We emphasize that this is a lower limit, as a few days of bad weather would be much more detrimental for finding these short-lived and lower luminosity transients compared to $\mathrm{SNe}$ Ia. This rate of $>2.3 \%$ relative to $\mathrm{SNe}$ Ia is consistent with the relative rate of $7 \% \pm 5 \%$ estimated by Perets et al. (2010).

The key to solving the mystery of the origin of "calciumrich gap" transients is their location. The remote locations can either be a red herring (due to small numbers) or the most important clue. Parenthetically, we remark that the two SNe Ia (SN 2006bt-Foley et al. 2010; PTF 10ops-Maguire et al. 2011) found farthest outside the main body of their host galaxies are also peculiar. Extremely deep late-time observations (e.g., with the Hubble Space Telescope) are needed to serve as a litmus test: detection of a dwarf satellite, super star cluster, or globular cluster would provide a direct clue about the environment, and nondetection would require a phoenix transient that rose from the ashes.

We thank Chuck Steidel and Ryan Trainor for target of opportunity observations of PTF 11 bij with Keck I. We are grateful to the staff of the Expanded Very Large Array for efficiently executing target of opportunity triggers. Excellent assistance was provided by the staffs of the various observatories where we obtained data (Lick, Keck, Palomar, WHT, and EVLA).

M.M.K. acknowledges support from the Hubble Fellowship and Carnegie-Princeton Fellowship. E.O.O. is supported by an Einstein Fellowship. H.B.P. is a CfA and Bikura prize fellow. The Weizmann Institute's participation in PTF is supported by grants to AGY from the Israel Science Foundation, the US-Israel Binational Science Foundation and the Minerva/ ARCHES Prize. A.V.F. and his group at UC Berkeley acknowledge generous financial assistance from Gary and Cynthia Bengier, the Richard and Rhoda Goldman Fund, the TABASGO Foundation, and National Science Foundation (NSF) grant AST0908886. KAIT was constructed and supported by donations from Sun Microsystems, Inc., the Hewlett-Packard Company, AutoScope Corporation, Lick Observatory, the NSF, the University of California, the Sylvia and Jim Katzman Foundation, and the TABASGO Foundation. Computational resources and data storage were contributed by NERSC, supported by U.S, DOE contract DE-AC02-05CH11231. P.E.N. acknowledges support from the U.S. DOE contract DE-FG02-06ER06-04. J.S.B. acknowledges support of an NSF-CDI grant 0941742, "Real-time Classification of Massive Time-series Data Streams.” M.S. acknowledges support from the Royal Society. L.B. acknowledges NSF grants PHY-11-25915 and AST-11-09174. D.C.L. acknowledges NSF grant AST-1009571.

Some of the data presented herein were obtained at the W. M. Keck Observatory, which is operated as a scientific partnership among the California Institute of Technology, the University of California, and the National Aeronautics and Space Administration; it was made possible by the generous financial support of the W. M. Keck Foundation. The Expanded Very Large Array is operated by the National Radio Astronomy Observatory, a facility of the NSF operated under cooperative agreement by Associated Universities, Inc. The WHT is operated on the island of La Palma by the Isaac Newton Group in the Spanish Observatorio del Roque de los Muchachos of the Instituto de Astrofisica de Canarias. All spectra are available in digital form at WISEREP (Yaron \& Gal-Yam 2012; http:www.weizmann.ac.il/astrophysics/wiserep/).

\section{REFERENCES}

Abazajian, K. N., Adelman-McCarthy, J. K., Agüeros, M. A., et al. 2009, ApJS, 182,543

Anderson, J. P., \& James, P. A. 2009, MNRAS, 399, 559

Arcavi, I., Gal-Yam, A., Kasliwal, M. M., et al. 2010, ApJ, 721, 777

Arnett, W. D. 1982, ApJ, 253, 785

Bildsten, L., Shen, K. J., Weinberg, N. N., \& Nelemans, G. 2007, ApJ, 662, L95

Bloom, J. S., Prochaska, J. X., Pooley, D., et al. 2006, ApJ, 638, 354

Boissier, S., \& Prantzos, N. 2009, A\&A, 503, 137

Cenko, S. B., Fox, D. B., Moon, D., et al. 2006, PASP, 118, 1396

Ferland, G. J., \& Persson, S. E. 1989, ApJ, 347, 656

Filippenko, A. V. 1997, ARA\&A, 35, 309

Filippenko, A. V., Chornock, R., Swift, B., et al. 2003, IAU Circ., 8159, 2

Filippenko, A. V., Li, W. D., Treffers, R. R., \& Modjaz, M. 2001, in ASP Conf. Ser. 246, IAU Colloq. 183, Small Telescope Astronomy on Global Scales, ed. B. Paczynski, W.-P. Chen, \& C. Lemme (San Francisco, CA: ASP), 121 Foley, R. J., Brown, P. J., Rest, A., et al. 2010, ApJ, 708, L61

Foley, R. J., Chornock, R., Filippenko, A. V., et al. 2009, AJ, 138, 376

Foley, R. J., Narayan, G., Challis, P. J., et al. 2010, ApJ, 708, 1748

Fruchter, A. S., Levan, A. J., Strolger, L., et al. 2006, Nature, 441, 463

Gal-Yam, A., Maoz, D., Guhathakurta, P., \& Filippenko, A. V. 2003, AJ, 125, 1087

Gal-Yam, A., Ofek, E. O., \& Shemmer, O. 2002, MNRAS, 332, L73

Hakobyan, A. A., Mamon, G. A., Petrosian, A. R., Kunth, D., \& Turatto, M. 2009, A\&A, 508, 1259

Hamuy, M., Phillips, M. M., Suntzeff, N. B., et al. 1996, AJ, 112, 2391

Heger, A., Fryer, C. L., Woosley, S. E., Langer, N., \& Hartmann, D. H. 2003, ApJ, 591,288

Howell, D. A. 2001, ApJ, 554, L193

Jha, S., Branch, D., Chornock, R., et al. 2006, AJ, 132, 189

Jordi, K., Grebel, E. K., \& Ammon, K. 2006, A\&A, 460, 339

Kasliwal, M. M., Ofek, E. O., Gal-Yam, A., et al. 2008, ApJ, 683, L29 
Kawabata, K. S., Maeda, K., Nomoto, K., et al. 2010, Nature, 465, 326 Law, N. M., Kulkarni, S. R., Dekany, R. G., et al. 2009, PASP, 121, 1395 Li, W., Chornock, R., Leaman, J., et al. 2011, MNRAS, 412, 1473

Li, W. D., Filippenko, A. V., Treffers, R. R., et al. 2000, in AIP Conf. Ser. 522, Cosmic Explosions, ed. S. S. Holt \& W. W. Zhang (Melville, NY: AIP), 103

Maguire, K., Sullivan, M., Thomas, R. C., et al. 2011, MNRAS, 418, 747

Mannucci, F., Della Valle, M., \& Panagia, N. 2006, MNRAS, 370, 773

Metzger, B. D. 2012, MNRAS, 419, 827

Metzger, B. D., Piro, A. L., Quataert, E., \& Thompson, T. A. 2009, arXiv:0908.1127

Mullan, B., Konstantopoulos, I. S., Kepley, A. A., et al. 2011, ApJ, 731, 93

Myers, A. T., Krumholz, M. R., Klein, R. I., \& McKee, C. F. 2011, ApJ, 735, 49

O'Connor, E., \& Ott, C. D. 2011, ApJ, 730, 70

Oke, J. B., Cohen, J. G., Carr, M., et al. 1995, PASP, 107, 375

Pastorello, A., Zampieri, L., Turatto, M., et al. 2004, MNRAS, 347, 74

Perets, H. B., Gal-yam, A., Crockett, R. M., et al. 2011, ApJ, 728, L36

Perets, H. B., Gal-Yam, A., Mazzali, P. A., et al. 2010, Nature, 465, 322

Phillips, M. M. 1993, ApJ, 413, L105

Prieto, J. L., Stanek, K. Z., \& Beacom, J. F. 2008, ApJ, 673, 999

Rau, A., Kulkarni, S. R., Law, N. M., et al. 2009, PASP, 121, 1334

Ruiter, A. J., Belczynski, K., Benacquista, M., \& Holley-Bockelmann, K. 2007, arXiv:0712.0847

Sahu, D. K., Anupama, G. C., Srividya, S., \& Muneer, S. 2006, MNRAS, 372 , 1315
Sahu, D. K., Tanaka, M., Anupama, G. C., et al. 2008, ApJ, 680, 580

Sand, D. J., Graham, M. L., Bildfell, C., et al. 2011, ApJ, 729, 142

Shafter, A. W., Darnley, M. J., Hornoch, K., et al. 2011, ApJ, 734, 12

Shen, K. J., Kasen, D., Weinberg, N. N., Bildsten, L., \& Scannapieco, E. 2010, ApJ, 715, 767

Sivanandam, S., Zabludoff, A. I., Zaritsky, D., Gonzalez, A. H., \& Kelson, D. D. 2009, ApJ, 691, 1787

Skrutskie, M. F., Cutri, R. M., Stiening, R., et al. 2006, AJ, 131, 1163

Slater, C. T., Bell, E. F., \& Martin, N. F. 2011, ApJ, 742, L14

Sullivan, M., Kasliwal, M. M., Nugent, P. E., et al. 2011, ApJ, 732, 118

Taubenberger, S., Valenti, S., Benetti, S., et al. 2009, MNRAS, 397, 677

Uomoto, A. 1986, ApJ, 310, L35

Valenti, S., Pastorello, A., Cappellaro, E., et al. 2009, Nature, 459, 674

van Dam, M. A., Bouchez, A. H., Le Mignant, D., et al. 2006, PASP, 118,310

van den Bergh, S. 1997, AJ, 113, 197

Waldman, R., Sauer, D., Livne, E., et al. 2011, ApJ, 738, 21

Werk, J. K., Putman, M. E., Meurer, G. R., et al. 2008, ApJ, 678, 888

Werk, J. K., Putman, M. E., Meurer, G. R., et al. 2010, AJ, 139, 279

Werk, J. K., Putman, M. E., Meurer, G. R., \& Santiago-Figueroa, N. 2011, ApJ, 735,71

Williams, B. F., Ciardullo, R., Durrell, P. R., et al. 2007, ApJ, 656, 756

Wizinowich, P. L., Chin, J., Johansson, E., et al. 2006, Proc. SPIE, 6272, 627209

Woosley, S. E., \& Kasen, D. 2011, ApJ, 734, 38

Woosley, S. E., \& Weaver, T. A. 1994, ApJ, 423, 371 